\title{
Sources of carbon and the evolution of the abundance of CNO elements
}

\author{
Y. C. Liang, G. Zhao, and J. R. Shi \\ Beijing Astronomical Observatory, Chinese Academy of Sciences, Beijing 100012, PR China \\ National Astronomical Observatories, Chinese Academy of Sciences, Beijing 100012, PR China
}

Received 29 March 2000 / Accepted 30 May 2001

\begin{abstract}
Using the standard infall model of Galactic chemical evolution, we explore the origin of carbon and calculate the abundance evolution of CNO elements for 8 different models of stellar nucleosynthesis yields. The results show that, in the early stage of the Galaxy, massive stars are the main producer of carbon, and that as our Galaxy evolves to the late stage, the longer lived intermediate- and low-mass stars play an increasingly important role, while at the same time, metal-rich Wolf-Rayet stars eject a significant amount of carbon into the ISM by radiative-driven stellar winds. However, from the present published nucleosynthesis yields we cannot distinguish whether the main source of carbon in the late Galactic stage is just the massive stars $\left(M>8 M_{\odot}\right)$ alone, or just the intermediate-, low-mass stars and $M \leq 40 M_{\odot}$ massive stars that do not go through the Wolf-Rayet stage. The ${ }^{12} \mathrm{C}(\alpha, \gamma){ }^{16} \mathrm{O}$ reaction rate is very important in the stellar nucleosynthesis calculations: a lower rate will give a higher yield of carbon. The contribution to nitrogen is dominated by intermediate- and low-mass stars, and the secondary source of massive stars cannot explain the observed [N/Fe] in metal-poor stars. Most of oxygen is produced by massive stars. The fact that a higher $\mathrm{O}$ abundance in metal-poor stars is derived from the $\mathrm{O}$ I 7771-7775 $\AA$ triplet than from the forbidden [O I] line at $6300 \AA$ poses a problem.
\end{abstract}

Key words. nuclear reactions, nucleosynthesis, abundances - stars: AGB - stars: supernova - stars: Wolf-Rayet Galaxy: abundance - Galaxy: evolution

\section{Introduction}

The abundance trends of the CNO elements are crucial to study the Galactic chemical evolution (hereafter GCE). These elements play important roles in the stellar interior as sources of opacity and energy production through the CNO cycle, and thus affect the star's lifetime, its position in the HR diagram, and its heavy-element yields. Carbon is especially important because it is the first element that is synthesized out of the primordial elements $(\mathrm{H}, \mathrm{He}, \mathrm{Li})$.

The main site of carbon synthesis has been a subject of argument for many years. Burbidge et al. (1957) suggested that carbon is provided by mass loss from red giants and supergiants. Iben \& Truran (1978) concluded from thermally pulsing models that intermediate-mass and highmass stars contribute carbon in roughly equal amounts. More recent theoretical and observational studies include the following.

Timmes et al. (1995) used the carbon yields of type II supernova (hereafter SN II) explosion from

Send offprint requests to: G. Zhao,

e-mail: zg@orion.bao.ac.cn
Woosley \& Weaver (1995) (hereafter WW) and the yields of intermediate-, low-mass stars (hereafter ILMS) from Renzini \& Voli (1981) (hereafter RV) to calculate the abundance evolution of carbon. They concluded that the ILMS contribute a significant amount of carbon to the interstellar medium (hereafter ISM). Chiappini et al. (1997) obtained similar results with their two-infall model. Oberhummer et al. (2000) reviewed the ILMS as the major producer of carbon. Kobulnicky \& Skillman (1998) measured the $\mathrm{C} / \mathrm{O}$ and $\mathrm{N} / \mathrm{O}$ ratios of three metal-poor galaxies with similar metallicities using HST ultraviolet and ground-based optical spectroscopy. Their results implied that the mechanisms of $\mathrm{C}$ and $\mathrm{N}$ production are coupled, and most of both $\mathrm{C}$ and $\mathrm{N}$ in galaxies originates in lowto intermediate-mass stars.

Prantzos et al. (1994) suggested that if the duration of the halo phase is as long as 1-2 Gyr, then massive stars with wind-driven mass loss could be the main carbon source during the whole galactic evolution. Gustafsson et al. (1999) adopted an analytical GCE model to calculate and compare the carbon abundance evolution in the Galactic disk $(-1.6<[\mathrm{Fe} / \mathrm{H}]<+0.2)$ and some 
irregular galaxies. They suggested that carbon enrichment is caused mainly by superwinds from metal-rich, massive stars. Karlsson et al. (1999) reported similar results. However, Gustafsson \& Ryde (2000) wrote that the source of carbon is still not clear. Henry et al. (2000) analyzed the behavior of the $\mathrm{N} / \mathrm{O}$ and $\mathrm{C} / \mathrm{O}$ abundance ratios as functions of metallicity in the Galactic and extragalactic $\mathrm{H}$ II regions. Their results showed that $\mathrm{C}$ and $\mathrm{N}$ originate from separate production sites and are decoupled from one another; and they suggested that massive stars $\left(M>8 M_{\odot}\right)$ dominate the production of carbon, while intermediatemass stars between 4 and $8 M_{\odot}$ dominate the nitrogen production. Carigi (2000) adopted different sets of yields to calculate the $\mathrm{C} / \mathrm{O}$ vs. Galactic age relation, and suggested massive stars to be the main contributor of carbon.

From calculated abundance gradients, Hou et al. (2000) argued that carbon comes from stellar winds of massive stars and ILMS. Goswami \& Prantzos (2000) using their new GCE model, concluded that the yields in WW are not enough to explain the observed carbon abundance, and that some other sources, Wolf-Rayet (hereafter $\mathrm{W}-\mathrm{R}$ ) stars or ILMS, are needed to explain the observed $[\mathrm{C} / \mathrm{Fe}]$ vs. $[\mathrm{Fe} / \mathrm{H}]$ curve. Garnett et al. (1999) measured the gas-phase abundance ratio $\mathrm{C} / \mathrm{O}$ in six $\mathrm{H}$ II regions in the spiral galaxies M 101 and NGC 2403, based on ultraviolet spectroscopy from the HST. These authors suggested that stellar winds in massive stars have an important effect on carbon and oxygen, and their analysis showed that the source of carbon depended on the assumed UV reddening function. When a flatter UV reddening $\left(R_{V}=5\right)$ was used, the data were consistent with the model that used the yields of massive stars from WW, while with a steeper value $\left(R_{V}=3\right)$ the data became more consistent with the model that used the yields of Maeder (1992) (hereafter M92).

In summary, it is still not clear whether carbon comes from the SNII explosions of massive stars, from stellar winds of W-R stars or from ILMS. In this paper we try to address this question from two aspects: (1) by using GCE model calculations with different sets of nucleosynthesis yields; (2) by using the observations of the large sample of dwarfs in our Galaxy as one of the main observational constraints.

For the first aspect, GCE modelling is an excellent tool to study the evolution of elements of the whole Galaxy and other galaxies. The main parameters for setting up such a model include the form of the infall, the star formation rate (SFR), the initial mass function (IMF) and the nucleosynthesis yields. The exponential form of infall (or Gaussian infall form: Chang et al. 1999 and Prantzos \& Silk 1998) has been found to be able to solve the Gdwarfs problem very well. The SFR and IMF can be determined from the observed present-day mass function (PDMF) (Pagel 1997). The most uncertain factor of GCE model is the nucleosynthesis yields, which largely decide the evolutionary trends of the elements. Different research groups have calculated the yields of stellar nucleosynthesis, but the results are rather different due to the use of different input parameters. And different yields will result in different evolution trends.

We set up a simple GCE model, like the one of Timmes et al. (1995), which fits some important observations. We choose the following different nucleosynthesis yields published recently in our calculations: for the RV yields of intermediate- and low-mass single stars, van den Hoek \& Groenewegen (1997) (hereafter VG), Marigo et al. (1996) (hereafter MBC1), Marigo et al. (1998) (hereafter MBC2) (hereafter, MBC represents for combined MBC1 and MBC2) and Marigo (2001) (hereafter M2K); for the WW yields of massive single stars, Nomoto et al. (1997a) (hereafter N97), M92 and Portinari et al. (1998) (hereafter PCB); for the yields of binaries through SN Ia explosion, Nomoto et al. (1997b). Then we compared and analyzed the different evolutionary behavior of the CNO elements, particularly $\mathrm{C}$, calculated on the basis of these different sets of yields.

In the early stage of our Galaxy, massive stars are the main producers of carbon. As our Galaxy evolves to a late stage, ILMS play an increasingly important role in the enrichment of the ISM due to their longer lifetimes. At the same time, the high metallicity W-R stars eject significant amounts of carbon into the ISM by radiative-driven stellar wind (the metallicity influences the opacities in the outer layers of massive stars, hence their mass loss rate through winds). Which is the main producer of carbon in the late stage of our Galaxy? We find an attempt to answer in the following way. We divide the massive stars into two mass ranges, $M>40 M_{\odot}$ and $M \leq 40 M_{\odot}$. And assume that the former does, and the latter does not, go through the W-R stage. We then compare the contributions by the W-R stars and ILMS to carbon in the late Galactic stage.

In the second part of our work, we pay particular attention to the observations of field dwarfs in our Galaxy. Their atmospheres are assumed to represent perfect records of the chemical compositions of the ISM at the time of their formation, and we have available the abundances for a large sample of field dwarfs in the solar neighborhood. In addition, the metallicity distribution of $\mathrm{G}$ dwarfs is very important, because the $\mathrm{G}$ dwarfs have lifetimes as long as the Galaxy, their metallicity distribution reflects the evolution of our Galaxy. The agemetallicity relation is significant, too, for reflecting the Galactic evolution.

This paper is organized as follows. In Sect. 2, we analyze the observational data. The GCE model and the relevant parameters are presented in Sect. 3. Different nucleosynthesis yields are analyzed in Sect. 4. Results of the analysis are given in Sects. 5-7. The main conclusions are summarized in Sect. 8.

\section{Observational data}

Observations of dwarf stars in the general field are very important to the evolution of chemical abundance as their atmospheres are thought to represent perfect records of 
the chemical compositions of the ISM at the time they formed.

\section{1. $[\mathrm{C} / \mathrm{Fe}]$ vs. $[\mathrm{Fe} / \mathrm{H}]$}

Carbon abundance can be calculated from the $\mathrm{CH}$ lines in the blue and ultraviolet (UV), the infrared CO emission, and a few red and near-infrared $\mathrm{C}_{\mathrm{I}}$ lines and $\left[\mathrm{C}_{\mathrm{I}}\right]$ forbidden lines. The [C I] line of low excitation potential being not so tightly dependent on NLTE considerations, the values derived from it are less dependent on the adopted stellar effective temperature than those from the high-excitation $\mathrm{C}$ I lines and $\mathrm{CH}$ lines. However, the small equivalent width $(E W)$ of the $[\mathrm{C} \mathrm{I}]$ line makes the measurement difficult. And the $[\mathrm{CI}]$ line at $\lambda 8727 \AA$ is supposed to be blended with a weak Fe I line. Both the $[\mathrm{C} \mathrm{I}]$ and $\mathrm{C}$ I lines become unmeasurably weak in metal-poor stars $([\mathrm{Fe} / \mathrm{H}]<-1)$.

The $\mathrm{CH}$ line in the UV can be used to obtain the $\mathrm{C}$ abundance of halo and disk stars. Laird (1985) published an extensive study of the $\mathrm{C}$ abundances of 116 field dwarfs, 10 faint field giants and 3 Hyades dwarfs in the range $-2.45<[\mathrm{Fe} / \mathrm{H}]<+0.5$. They suggested that the $[\mathrm{C} / \mathrm{Fe}]$ were solar in the whole $[\mathrm{Fe} / \mathrm{H}]$ range of the samples. Tomkin et al. (1986) analyzed the spectra of 32 halo dwarfs, and derived $[\mathrm{C} / \mathrm{Fe}] \approx-0.2$, independent of metallicity for stars with $[\mathrm{Fe} / \mathrm{H}]>-2$. And their data showed a rise of $\mathrm{C}$ abundance in more metal-poor stars: $[\mathrm{C} / \mathrm{Fe}] \approx$ +0.2 at $[\mathrm{Fe} / \mathrm{H}] \approx-2.5$. Carbon et al. (1987) derived the $\mathrm{C}$ abundances of 83 halo dwarfs, which showed that $[\mathrm{C} / \mathrm{Fe}]$ is constant over the range of $-2.5<[\mathrm{Fe} / \mathrm{H}] \leq-0.75$, and they found an upturn at the very low metallicity value. Wheeler et al. (1989) reviewed these observed trends of $[\mathrm{C} / \mathrm{Fe}]$ vs. $[\mathrm{Fe} / \mathrm{H}]$.

Tomkin et al. (1992) derived the $\mathrm{C}$ abundances of halo and disk stars using the $\mathrm{C}_{\mathrm{I}} \lambda 9100 \AA$ line. Their results showed that the $\mathrm{C}$ abundances are +0.2 higher than those derived from the $\mathrm{CH}$ lines. Andersson \& Edvardsson (1994) (using [C I] $8727 \AA$ ), Tomkin et al. (1995) (using $\mathrm{C}_{\mathrm{I}} 7100 \AA$ ) and Gustafsson et al. (1999) (using [C I] $8727 \AA$ ) obtained the $\mathrm{C}$ abundances for large samples of disk dwarfs (comprising 85, 105 and 80 stars respectively, with some in common). Their results showed that $[\mathrm{C} / \mathrm{Fe}]$ decreased with increasing $[\mathrm{Fe} / \mathrm{H}]$ in the Galactic disk region. Using $\mathrm{CH}$ and $\mathrm{C}_{2}$ lines, Carretta et al. (2000) analyzed a sample of 19 stars, and reanalyzed the stars of Tomkin et al. (1992) and Edvardsson et al. (1993). Their results showed that $[\mathrm{C} / \mathrm{Fe}]$ is roughly solar in the whole metallicity range of $[\mathrm{Fe} / \mathrm{H}]>-2.5$.

Considering the large scatter, we choose to take all the above-mentioned observations of $\mathrm{C}$ abundance as observational constraints on our GCE calculations.

\section{2. $[\mathrm{O} / \mathrm{Fe}]$ vs. $[\mathrm{Fe} / \mathrm{H}]$}

Oxygen is the third most abundant element in the universe, and it is very important for the study of GCE.
In general, oxygen is produced by massive stars through SN II explosions, like other $\alpha$ elements. Thus, the observations of oxygen, especially in metal-poor region, give a measure of the contribution by massive stars. Some parameters required in GCE calculations can be determined by the observed $[\mathrm{O} / \mathrm{Fe}]$ in the stars (Prantzos et al. 1994).

The oxygen abundance of stars can be derived from the O I $\lambda \lambda 7771-7775 \AA$ triplet, the [O I] $\lambda 6300 \AA$ forbidden line and $\mathrm{OH}$ lines in the UV region. In main-sequence, metal-poor stars, the [O I] lines are extremely weak and are difficult to use for this purpose. Nonetheless, sensitive detectors, high-resolution, and long exposures have enabled several teams (Spite \& Spite 1991; Nissen \& Edvardsson 1992) to measure the $E W_{\mathrm{s}}$ of some dwarfs with $[\mathrm{Fe} / \mathrm{H}] \leq-0.1$. However, the $[\mathrm{O}$ I] line becomes too weak to measure at $[\mathrm{Fe} / \mathrm{H}] \leq-1.3$, and to provide useful information on the trend of $[\mathrm{O} / \mathrm{Fe}]$ vs. $[\mathrm{Fe} / \mathrm{H}]$. Thus, for the metal-poor stars, the O I triplet lines are the preferred choice in dwarfs because they are of measurable strength for a wide range of stellar temperatures and they lie in a fairly clear portion of the stellar spectrum. Molecular $\mathrm{OH}$ lines in the near-ultraviolet at $\lambda \lambda 3060-3200 \AA$, are observed in both giants and dwarfs with $T_{\text {eff }} \leq 6500 \mathrm{~K}$ $(\mathrm{OH}$ lines are destroyed at higher temperatures), and can be used to derive the $\mathrm{O}$ abundance.

An interesting result is that there is a systematic difference of about 0.5 dex between the $\mathrm{O}$ abundances derived from the permitted and forbidden lines. The [O I] 6300 line provides about $0.4-0.5$ dex of the lower $[\mathrm{O} / \mathrm{Fe}]$ values in metal-poor stars (e.g. Spite \& Spite 1991; Spiesman \& Wallerstein 1991; Fulbright \& Kraft 1999; Nissen et al. 2000 for dwarfs; Barbuy 1988; Barbuy \& Erdelyi-Mendes 1989; Sneden et al. 1991 for giants). The O I triplet and $\mathrm{OH}$ lines, on the other hand, produce the higher $[\mathrm{O} / \mathrm{Fe}]$ values, reaching +1.0 at $[\mathrm{Fe} / \mathrm{H}] \approx-3$ (Abia \& Rebolo 1989; King 1994; Nissen et al. 1994; Israelian et al. 1998; Boesgaard et al. 1999; Mishenina et al. 2000). Perhaps the higher $\mathrm{O}$ abundance obtained from the O I triplet was caused by some NLTE effect (Mishenina et al. 2000 and references therein). Kiselman (1991) has performed a NLTE analysis of O I $\lambda 7774 \AA$ and found significant NLTE corrections, up to 0.4 dex. However, Takeda (1994) found the NLTE effects to be small in metal-poor stars. Another source of discrepancy may be the fact that the $\mathrm{O} / \mathrm{Fe}$ ratio is very sensitive to $T_{\text {eff }}$ (Nissen \& Edvardsson 1992). King (1993) determined a reduction in the temperature of subgiants of 150-200 K, and obtained similar O abundances from the $\lambda 7774 \AA$ and $\lambda 6300 \AA$ lines. King \& Boesgaard (1995) obtained the $\mathrm{O}$ abundances from both the $[\mathrm{OI}] \lambda 6300 \AA$ and $\mathrm{O}$ I $\lambda 7774 \AA$ lines for a sample of metal-rich $\mathrm{F}$ and $\mathrm{G}$ dwarfs. They found that for $T_{\text {eff }} \leq$ $6200-6300 \mathrm{~K}$, no systematic difference exists in the abundances determined from the $\lambda \lambda 6300$ and $7774 \AA$ lines. For $T_{\text {eff }} \geq 6200-6300 \mathrm{~K}$, however, the $\lambda 7774 \AA$ abundance is substantially higher than the $\lambda 6300 \AA$ abundance.

Thus, many papers have demonstrated that there is a systematic difference between the values of $\mathrm{O}$ abundance derived from the forbidden and permitted lines. Possible 
reasons are as follows. The excitation potential of the O I triplet is very high $(9.15 \mathrm{eV})$, the line is formed deep down in the atmosphere, and the abundance determination has a large error; the near-UV OH lines at 3060-3200 $\AA$ are sensitive to the gas pressure and electron density in the model atmosphere. In contrast, the [O I] line is roughly half as sensitive as the $\mathrm{OH}$ and $\mathrm{O}$ I lines, and is insensitive to changes in the ratio of $\alpha$-element to iron. Also, the $\mathrm{OH}$ lines are very crowded in the UV region, which makes their measurement difficult.

Nissen et al. (2000) obtained the O abundances of 13 dwarfs and subgiants using data from the ESO VLT. They suggested that the $[\mathrm{O} / \mathrm{Fe}]$ is nearly constant at $\approx+0.4$ for $[\mathrm{Fe} / \mathrm{H}]$ below $\approx-1.0$. Maciel (2000) suggested that $[\mathrm{O} / \mathrm{Fe}]$ is not higher than +0.4 according to the radial $\mathrm{O} / \mathrm{H}$ abundance gradients in $\mathrm{H}$ II regions, hot stars and $\mathrm{PNe}$, and $[\mathrm{Fe} / \mathrm{H}]$ gradients from open cluster stars.

From the foregoing discussion and considering the similar behavior between $\mathrm{O}$ and $\alpha$ elements $([\alpha / \mathrm{Fe}]$ is about +0.4 in metal-poor stars, see Zhao \& Magain 1990 and Fuhrmann 1998), we use the lower O abundances derived from the $[\mathrm{OI}]$ line as observational constraints on our GCE calculations. And we will specially discuss the higher abundances in metal-poor stars obtained from the $\mathrm{O}$ I triplet and $\mathrm{OH}$ lines in Sect. 8. Because the observed O abundances of metal-poor dwarfs are not enough to reflect the abundance evolution, we have supplemented our data with some metal-poor giants.

\section{3. $[\mathrm{N} / \mathrm{Fe}]$ vs. $[\mathrm{Fe} / \mathrm{H}]$}

The N I line can be used in the abundance determination of nitrogen, but this can only be done for reasonably metalrich stars. Clegg et al. (1981) obtained the $\mathrm{N}$ abundances of 20 disk stars $(-0.9<[\mathrm{Fe} / \mathrm{H}]<+0.4)$ using the $\mathrm{N}$ I line, and suggested that $[\mathrm{N} / \mathrm{Fe}] \approx 0.0$. For halo stars, some results have been derived from NH lines in the UV region. Tomkin \& Lambert (1984), Laird (1985) and Carbon et al. (1987) obtained similar results, $[\mathrm{N} / \mathrm{Fe}] \approx 0.0$, irrespective of metallicity in the range $-2<[\mathrm{Fe} / \mathrm{H}]<+0.3$. Indeed, there are still some difficulties in deriving reliable $\mathrm{N}$ abundance from the NH bands at $\lambda \lambda 3360$ and $3370 \AA$. The difficulties are (a) uncertainties in the continuous opacities at these wavelengths, (b) severe atomic-line contamination of the $\mathrm{NH}$ features, (c) uncertainty in the dissociation energy of this molecule. (d) the crowdedness of the lines in the UV region. Also, it is very difficult to derive the $\mathrm{N}$ abundance from the CN bands at $\lambda \lambda 4200$ or $3800 \AA$. Possible reasons are that the dissociation energy of $\mathrm{CN}$ is not known to better than a factor of 2 , and that the determination of $\mathrm{N}$ abundance from $\mathrm{CN}$ depends on a prior determination of reliable $\mathrm{C}$ abundance from other features, and that the $\mathrm{CN}$ bands are extremely weak in very metal-poor stars. A detailed discussions can be found in Wheeler et al. (1989).

\subsection{Table of observations}

Table 1 summarises the sources of the observations of carbon, nitrogen and oxygen. Most of the objects are dwarfs and subgiants; a few metal-poor giants provide additional $\mathrm{O}$ abundance data derived from the $[\mathrm{OI}]$ $\lambda 6300 \AA$ forbidden line.

\subsection{The age-metallicity relation and the metallicity distribution of $G$ dwarfs}

The age-metallicity relation for the solar neighborhood is one of the important observational constraints; it shows the $[\mathrm{Fe} / \mathrm{H}]$-represented metallicity as a function of the star's age. The observational data and scatter are taken from Carlberg et al. (1985), Meusinger et al. (1991) and Edvardsson et al. (1993) (their Tables 14 and 15).

Another important observational constraint is the metallicity distribution of long-lived G-type stars. Because these stars have long main-sequence lifetimes of about 15 Gyr, comparable to the estimated age of the Galaxy, they represent a sample of stars which have not been removed by stellar evolution. The observational metallicity distribution of G dwarfs is taken from Chang et al. (1999). The solar abundances of CNO elements are taken from Grevesse \& Sauval (1998).

\section{Model of galactic chemical evolution}

A general, standard infall GCE model, namely, a multizone model for GCE without mass exchange among the zones is adopted in our present study. The rate of change of the surface mass density of isotope $i$ in every zone in the gas at a given Galactocentric radius $r$ and time $t$ is

$$
\begin{aligned}
& \frac{\mathrm{d} \sigma_{\mathrm{g}, i}(r, t)}{\mathrm{d} t}=-X_{i}(r, t) \Psi(r, t) \\
& +\int_{M_{\mathrm{l}}}^{M_{\mathrm{b} 1}} X_{i}\left(r, t-\tau_{\mathrm{M}}\right) \Psi\left(r, t-\tau_{\mathrm{M}}\right) \Phi(M) \mathrm{d} M \\
& +\beta \int_{M_{\mathrm{bl}}}^{M_{\mathrm{bu}}} \Phi\left(M_{\mathrm{b}}\right)\left[\int_{\mu_{\mathrm{m}}}^{0.5} f(\mu) X_{i}\left(r, t-\tau_{\mathrm{M}_{2}}\right)\right. \\
& \left.\times \Psi\left(r, t-\tau_{\mathrm{M}_{2}}\right) \mathrm{d} \mu\right] \mathrm{d} M_{\mathrm{b}} \\
& +(1-\beta) \int_{M_{\mathrm{bl}}}^{M_{\mathrm{bu}}} X_{i}\left(r, t-\tau_{M}\right) \Psi\left(r, t-\tau_{M}\right) \Phi(M) \mathrm{d} M \\
& +\int_{M_{\mathrm{bu}}}^{M_{\mathrm{u}}} X_{i}\left(r, t-\tau_{\mathrm{M}}\right) \Psi\left(r, t-\tau_{\mathrm{M}}\right) \Phi(M) \mathrm{d} M \\
& +\left[\frac{\mathrm{d} \sigma_{i}(r, t)}{\mathrm{d} t}\right]_{\mathrm{inf}},
\end{aligned}
$$

where the first term describes the disappearance of isotope $i$ due to new star formation, the second term describes the enrichment due to stellar wind mass loss of 
Table 1. Observational data from different references and the line sources.

\begin{tabular}{|c|c|c|c|c|c|}
\hline References & $\begin{array}{l}\mathrm{C} \\
\text { line, wavelength }(\AA)\end{array}$ & $\begin{array}{l}\mathrm{N} \\
\text { line, wavelength }(\AA)\end{array}$ & $\begin{array}{l}\mathrm{O} \\
\text { line, wavelength }(\AA)\end{array}$ & {$[\mathrm{Fe} / \mathrm{H}]$} & Numbers of stars \\
\hline 1 & $\mathrm{C}$ I, $[\mathrm{C}$ I $], \mathrm{CH}$ & N I 8683 & O г $6158,[$ O I] 6300 & {$[-0.90,+0.40]$} & 20 \\
\hline 2 & [C I] 8727 & & & {$[-1.00,+0.25]$} & 85 \\
\hline 3 & [C I $] 8727$ & & & {$[-1.06,+0.26]$} & 80 \\
\hline 4 & C I 7100 & & & {$[-0.80,+0.20]$} & 105 \\
\hline 5 & C I 9100 & & O 7770 triplet & {$[-3.00,-0.80]$} & 34 \\
\hline 6 & $\mathrm{CH} 4300$ & NH 3360 & & {$[-2.45,+0.50]$} & 116 \\
\hline 7 & CH 4300 & & & {$[-2.60,-0.70]$} & 32 \\
\hline 8 & CH 4300 & NH 3360 & & {$[-3.20,-1.50]$} & 83 \\
\hline 9 & $\mathrm{CH} \mathrm{C} 2$ & $\mathrm{CN}$ & $\mathrm{O}$ I, [O I] & {$[-2.61,+0.12]$} & 19 (some giants) \\
\hline 10 & & NH 3360 & & {$[-2.30,-0.30]$} & 14 \\
\hline 11 & & & [O I] 6300 & {$[-1.60,+0.30]$} & 7 \\
\hline 12 & & & [O I] 6300 & $-1.74,-1.43$ & 2 \\
\hline 13 & & & [O I] 6300 & {$[-0.80,+0.30]$} & 23 \\
\hline 14 & & & [O I] 6300 & $-2.84,-2.31$ & 2 \\
\hline 15 & & & [O I] 6300 & {$[-1.80,-0.70]$} & 13 \\
\hline 16 & & & O I 7770 triplet & {$[-1.03,+0.26]$} & 86 \\
\hline 17 & & & O I 7770 triplet & {$[-1.00,+0.10]$} & 90 \\
\hline 18 & & & O I 7770 triplet & $-1.84,-2.08$ & 2 \\
\hline 19 & & & O I 7770 triplet & {$[-2.50,-0.50]$} & 14 (some giants) \\
\hline 20 & & & O I 7770 triplet & {$[-2.72,-0.47]$} & 14 \\
\hline 21 & & & O I 7770 triplet & -3.00 & 1 \\
\hline 22 & & & O I 7770 triplet & {$[-3.50,-0.20]$} & 30 \\
\hline 23 & & & $\mathrm{OH}$ & {$[-3.00,-0.30]$} & 24 \\
\hline 24 & & & $\mathrm{OH}$ & {$[-3.02,-0.50]$} & 24 \\
\hline 25 & & & OH $3138-3155$ & {$[-3.20,-1.80]$} & 9 \\
\hline 26 & & & $\mathrm{OH}, \mathrm{CO}$ & -1.22 & 1 \\
\hline 27 & & & [O I] 6300 & {$[-1.20,-0.10]$} & 24 (some giants) \\
\hline 28 & & & [O I] 6300 & {$[-3.00,-1.10]$} & 20 (giants) \\
\hline 29 & & & [O I] 6300 & {$[-2.35,+0.37]$} & 18 (giants) \\
\hline 30 & & & [O I] 6300 & {$[-2.88,-1.80]$} & 10 (giants) \\
\hline
\end{tabular}

1. Clegg et al. (1981); 2. Andersson \& Edvardsson (1994); 3. Gustafsson et al. (1999); 4. Tomkin et al. (1995); 5. Tomkin et al. (1992); 6. Laird (1985); 7. Tomkin et al. (1986); 8. Carbon et al. (1987); 9. Carretta et al. (2000); 10. Tomkin \& Lambert (1984); 11. Spite \& Spite (1991); 12. Spiesman \& Wallerstein (1991); 13. Nissen \& Edvardsson (1992); 14. Fulbright \& Kraft (1999); 15. Nissen et al. (2000); 16. Edvardsson et al. (1993); 17. Chen et al. (2000); 18. Beveridge \& Sneden (1994); 19. Mishenina et al. (2000); 20. Boesgaard \& King (1993); 21. King (1994; 22. Abia \& Rebolo (1989); 23. Israelian et al. 1998; 24. Boesgaard et al. (1999); 25. Nissen et al. (1994); 26. Balachandran \& Carney (1996); 27. Barbuy \& Erdelyi-Mendes (1989); 28. Barbuy (1988); 29. Gratton \& Ortolani (1986); 30. Sneden et al. (1991).

low mass single stars, the third term represents the rate of enrichment due to binary systems that undergo SN Ia explosions. The free parameter $\beta$ is the amplitude factor of type Ia supernova (hereafter SN Ia) explosion and $f(\mu)$ is the binary distribution function. The fourth term represents the enrichment due to single stars or binary systems that do not undergo type Ia events in the mass interval $M_{\mathrm{bl}}$ to $M_{\mathrm{bu}}$. The fifth term represents the enrichment rate due to massive stars that become SN II explosions with masses between $M_{\mathrm{bu}}$ to $M_{\mathrm{u}}$. The last term represents the infall of the primordial material. $X_{i}(r, t)$ is the elemental yield from nucleosynthesis (see Sect. 4). More details have been given by Matteucci \& François (1989) and Timmes et al. (1995).

- $\Psi(r, t)$ (SFR): Analytical prescriptions for the SFR can be obtained from the observations. Schmidt (1959) suggested that the SFR is related to the surface mass density of interstellar gas $\sigma_{\mathrm{g}}$. Subsequent studies showed that the SFR depends not only on the surface mass density of gas but also on the total surface mass density of the ISM (Talbot \& Arnett 1975; Dopita \& Ryder 1994; Timmes et al. 1995; Chiappini et al. 1997; Dwek 1998).

We adopt a similar formula to that of Timmes et al. (1995):

$\Psi(r, t)=\nu \sigma_{\text {tot }}(r, t)\left[\frac{\sigma_{\text {gas }}(r, t)}{\sigma_{\text {tot }}(r, t)}\right]^{n} M_{\odot} \mathrm{pc}^{-2} \mathrm{Gyr}^{-1}$,

where $n$ is in the range 1-2 (Timmes et al. 1995), which we fix at 1.5 throughout our calculation, $\nu$ is the efficiency factor in unit $\mathrm{Gyr}^{-1}$, which can be adjusted around the value 1.0 .

- $\Phi(M)$ (IMF): For the solar neighborhood, the IMF is given in a series of papers. The first formula was applied by Salpeter (1955), who suggested the single power-law form: $\Phi(M) \sim M^{-(1+x)}(x=1.35)$, normalized to 1 over a given mass range. Then Scalo (1986) suggested that the 
power law should become steeper for $M \geq 1 M_{\odot}, x=1.7$. Following them, more authors suggested that IMF should flatten at the low mass end, for example Kroupa et al. (1993). Dwek (1998) analyzed these results, and gave a similar formula as Kroupa et al. (1993) (for three mass ranges). An important issue for the IMF is it being "topheavy", meaning that the formation frequency of massive stars in the early Galactic stage was higher than at the present. Recently, the question of a changing IMF as our Galaxy evolves has been examined. Chiappini et al. (2000) found that the metallicity distribution of $\mathrm{G}$ dwarfs is well explained by a constant IMF, rather than by a changing one. Martinelli \& Matteucci (2000) found that, with a changing IMF, even though the metallicity distribution of $\mathrm{G}$ dwarfs could be reproduced, other important observations of $[\mathrm{O} / \mathrm{Fe}]$ would then not fit so well.

In our calculation, we adopt the formula of Dwek (1998):

$$
\begin{aligned}
\Phi(M) & =C_{1} & & \text { for } 0.1 \leq M / M_{\odot} \leq 0.3 \\
& =C_{2} M^{-1.6} & & \text { for } 0.3 \leq M / M_{\odot} \leq 1 \\
& =C_{3} M^{-2.6} & & \text { for } 1 \leq M / M_{\odot} \leq 100
\end{aligned}
$$

where $C_{1}, C_{2}$ and $C_{3}$, the normalized coefficients, are determined from the normalization $\int_{M_{1}}^{M_{\mathrm{u}}} M \Phi(M) \mathrm{d} M=1$, with $M_{1}=0.1 M_{\odot}$ and $M_{u}=100 M_{\odot}$. In fact, the bulk of chemical enrichment is caused by the stars with $M \geq 1 M_{\odot}$. Thus, it is meaningful to fix the fraction $\zeta$ of the total stellar mass distributed in stars above $1 M_{\odot}$ $\left(M_{1}\right)$, which is equivalent to fixing $M_{1}$, namely,

$$
\begin{aligned}
\int_{M_{1}}^{M_{\mathrm{u}}} M \Phi(M) \mathrm{d} M & =\int_{M_{1}}^{M_{1}} M \Phi(M) \mathrm{d} M+\int_{M_{1}}^{M_{\mathrm{u}}} M \Phi(M) \mathrm{d} M \\
& =\int_{M_{1}}^{M_{1}} M \Phi(M) \mathrm{d} M+\zeta=1 .
\end{aligned}
$$

Pagel (1997) explained this method in detail. In our calculations for different sets of yields, we adjust $\zeta$ so that the results give a good fit to the observations, especially the metallicity distribution of $\mathrm{G}$ dwarfs.

-Infall: The infall rate is generally taken to follow an exponent law (Matteucci \& François 1989; Timmes et al. 1995) or Gaussian form (Prantzos \& Silk 1998; Chang et al. 1999). We adopt the specific form of Timmes et al. (1995), but with $\sigma_{\odot}=55 M_{\odot} \mathrm{pc}^{-2}$ (Sackett 1997).

-Main-sequence lifetimes $\tau_{\mathrm{M}}$ : The main-sequence lifetimes of stars are taken from Schaller et al. (1992), which have been used generally in GCE calculations, such as Timmes et al. (1995) and Prantzos \& Silk (1998).

\section{Stellar yields and nucleosynthesis}

The stars are divided into binary and single stars. We only consider SN Ia explosion for the binary stars, and divide the single stars into two groups: intermediate-, lowmass stars $\left(0.9-8 M_{\odot}\right.$, or $0.9-6 M_{\odot}$ in case of strong overshooting) and massive stars $\left(M>8 M_{\odot}\right.$, or $M>$ $6 M_{\odot}$ in case of strong overshooting).

\subsection{Intermediate-, low-mass single stars}

The first detailed nucleosynthesis calculation for ILMS was made by $\operatorname{RV}\left(M=1-8 M_{\odot}, Z=0.004\right.$, $0.02)$. Recently, VG extended the calculation to $M=$ 0.8-8.0 $M_{\odot}$ and $Z=0.001,0.004,0.008,0.02,0.04$. Forestini \& Charbonnel (1997) calculated the evolution of stars with $M=3,4,5,6,7 M_{\odot}$ and $Z=0.005,0.02$. MBC calculated the evolution of stars with $M=0.819-5.0 M_{\odot}$ and $Z=0.008,0.02$ for the case of strong overshooting, which means that the stars evolve rapidly so that the upper mass limit of ILMS is changed from the standard value of $8 M_{\odot}$ to $6 M_{\odot}$. M2K gave a new set of stellar yields (for $M=0.817-5.0 M_{\odot}$ and $\left.Z=0.004,0.008,0.02\right)$ based on the results of an updated evolution calculation.

In our calculations, we choose the results of RV (their $\alpha=1.5, \eta=0.333$ case), $\mathrm{VG}, \mathrm{MBC}$ and $\mathrm{M} 2 \mathrm{~K}$ as the yields of ILMS for their wide mass ranges and metallicitydependent results. RV only gave results of $3.25 M_{\odot}<$ $M<8 M_{\odot}$ for the case of $\alpha=1.5, \eta=0.333$. In fact, the hot-bottom burning (HBB) process is not important for low mass stars, but is very significant for intermediate mass stars above $4 M_{\odot}$. So we choose the results with $\alpha=$ 0.0 for the lower mass stars. M2K calculated three sets of yields with $\alpha=1.68,2.00,2.50$ for stars with $M \geq 3.5 M_{\odot}$. $\alpha=2.50$ is too high to fit the observations (M2K), so we only consider the results for $\alpha=1.68,2.00$.

\subsection{Binary stars through SN la explosions}

Type Ia SN explosions are assumed to occur in close binary systems (Whelan \& Iben 1973). In this model, the explosion is caused by a carbon-deflagration of the material accreting on the degenerate white dwarf (Nomoto et al. 1997b), and the ejecta is dominated by the ${ }^{56} \mathrm{Fe}$ isotope. In this paper, the nucleosynthesis yields of SN Ia explosions are taken from the classical W7-model of Nomoto et al. (1997b), in which $\sim 0.613 M_{\odot}$ of Fe is produced.

\subsection{Massive single stars}

A star in the mass range of $8-10 M_{\odot}\left(\right.$ or $6-8 M_{\odot}$ in the case of strong overshooting) generally develops a degenerate $\mathrm{O}-\mathrm{Ne}-\mathrm{Mg}$ core after C-burning and eventually explodes as an electron capture supernova, leaving a neutron star of $1.3 M_{\odot}$ as a remnant and expelling a very small quantity of heavy elements.

A star with a higher initial mass will end its life with an Fe-core collapse type II SN explosion. Some authors, including WW, N97, M92 and PCB, calculated the stellar nucleosynthesis yields for such stars either with or without mass loss through wind. The results differ because of different choices of the parameters. We have carefully compared their parameters and results in 
Liang \& Zhao (2001). Some of the parameters adopted by them are listed in Table 2, including the stellar mass, metallicity, and ${ }^{12} \mathrm{C}(\alpha, \gamma){ }^{16} \mathrm{O}$ reaction rate.

Limongi et al. (2000) calculated the presupernova nucleosynthesis of massive stars with $M=13,15,20,25 M_{\odot}$ and $Z=0.02$, and the explosive nucleosynthesis of stars with $Z=10^{-3}, 0$. They did not give the yields of stars with $M>25 M_{\odot}$, which are very important for tracing the chemical evolution. And they did not give the explosive nucleosynthesis of stars with $Z=0.02$. So we did not use their results in our calculation of the abundance evolution of CNO elements in the present paper.

\subsubsection{Yields calculated by WW}

WW calculated, for a large mass range, the metallicitydependent yields of SN II explosion, and the results have been widely used in GCE models (e.g. Timmes et al. 1995; Chiappini et al. 1997)

Timmes et al. (1995) suggested that the results of abundance evolution would be better if the Fe yields of WW is reduced by a factor of 2 . Samland (1998) suggested that the actual Fe yield of SN II explosion should be $0.046 M_{\odot}$, approximately a factor of 2 less than the WW value. Carigi (1994) used $0.075 M_{\odot}$ as the Fe yields of SN II explosion in their GCE model. Chieffi et al. (1998) calculated the nucleosynthesis of SN II explosion for a $25 M_{\odot}$ star, and got an Fe yield of about $0.075 M_{\odot}$. SN 1987A, for an initial main sequence mass of $20 M_{\odot}$, ejected $0.075 M_{\odot}$ of $\mathrm{Fe}$, while for the same initial mass, WW got $0.151 M_{\odot}$, again twice as large. Because of these findings we reduced the results of WW by a factor of 2 in our calculations.

\subsubsection{Yields given by $\mathrm{N} 97$}

N97 extended the calculations of Thielemann et al. (1996) to include $13-70 M_{\odot}$, solar metallicity stars. We should note that N97 used the evolution of the He core instead that of the entire star. They used a relation $M\left(M_{\alpha}\right)$ to transfer the result for a helium core of mass $M_{\alpha}$ to that of a star of initial mass $M$ (Sugimoto \& Nomoto 1980). This can be understood since the total ejecta of star must be the sum of the contributions of both the He-core and the envelope of mass $M-M_{\alpha}$. (Thomas et al. 1998).

\subsubsection{Yields given by $\mathrm{M} 92$ and $\mathrm{PCB}$}

Both M92 and PCB considered radiative-driven stellar wind in their calculations, which was important for massive stars, especially the W-R stars. The synthesized elements of $\mathrm{C}, \mathrm{N}$ and $\mathrm{O}$, in particular, $\mathrm{C}$, can be ejected into the ISM in stellar wind.

How to choose the yields of W-R stars? Massey et al. (1995) studied the massive stars both in the general field and associations of the Magellanic Clouds. Their data showed that stars with initial masses $M>30 M_{\odot}$ evolved through the W-R phase in the Large Magellanic Cloud (LMC); while the statistics of the Small Magellanic Cloud (SMC) are consistent with a somewhat higher mass limit of possibly $50 M_{\odot}$. Conti (1995) suggested that the W$\mathrm{R}$ stars are highly evolved, luminous, hot, and (mostly) He-burning descendants of the most massive stars in the solar neighborhood, with masses $M \geq 35 M_{\odot}$. In the present paper, we follow the suggestions of Maeder \& Conti (1994) and the references therein and assume that the stars with $M>40 M_{\odot}$ will evolve through the W$\mathrm{R}$ stage. So we divide the massive stars into two mass ranges, $M \leq 40 M_{\odot}$ and $M>40 M_{\odot}$, in our discussion on the source of carbon. For the upper mass limit of massive stars, we choose $100 M_{\odot}$, following the suggestion of Leitherer (1995), and the usual practice with the upper mass limit of IMF used in many GCE models (e.g. PCB, Prantzos \& Silk 1998)

M92 did not give Fe yields. When we use the M92 set of yields, we choose the following values: $0.15 M_{\odot}$ for stars with initial main sequence mass $M \leq 14 M_{\odot}$, $0.075 M_{\odot}$ for $14 M_{\odot}<M \leq 40 M_{\odot}$, and $0.15 M_{\odot}$ for $M>40 M_{\odot}$. These choices are based on the following considerations. In the case of SN 1987A (20 $M_{\odot}$ during the main-sequence stage), the light curve, powered by the decay of ${ }^{56} \mathrm{Ni}$ and ${ }^{56} \mathrm{Co}$, gives a determination of the produced ${ }^{56} \mathrm{Fe}$ of $0.075 M_{\odot}$, and for SN 1993J (main-sequence mass $14 M_{\odot}$ ), one of $0.15 M_{\odot}$. (Thielemann et al. 1996). Carigi (1994) adopted $0.075 M_{\odot}$ of Fe for a SN II explosion and $0.15 M_{\odot}$ for a SN Ib explosion. Since SN Ib takes place in W-R stars, and since we assume that the stars with $M>40 M_{\odot}$ will undergo the $\mathrm{W}$-R stage, so we use the value $0.15 M_{\odot}$ in such cases.

M92 only gave the yield of $\mathrm{N}$ ejected in wind, and not the final value. Thus the $\mathrm{N}$ yields used in our calculations are lower limits.

\section{Results and analyses}

We set up a standard infall model of GCE to map out the abundance evolution of carbon, nitrogen and oxygen in the solar neighborhood. We choose the general formulae of SFR and IMF, and slightly adjust them by changing $\nu$ and $\zeta$ respectively. The 8 sets of nucleosynthesis yield, described in Sect. 4 are then used to set up 8 specific models. For each model, we try to fit the observational age-metallicity relation, the metallicity distribution of $\mathrm{G}$ dwarfs and the $[\mathrm{O} / \mathrm{Fe}]$ vs. $[\mathrm{Fe} / \mathrm{H}]$ relation. The calculated results will then be discussed: the $[\mathrm{C} / \mathrm{Fe}]$ vs. $[\mathrm{Fe} / \mathrm{H}]$ relation, in this section. the $[\mathrm{N} / \mathrm{Fe}]$ vs. $[\mathrm{Fe} / \mathrm{H}]$ and $[\mathrm{O} / \mathrm{Fe}]$ vs. $[\mathrm{Fe} / \mathrm{H}]$ relations in Sects. 6 and 7 , respectively.

\section{1. $V G+W W$}

In this model labelled ( $\mathrm{VG}+\mathrm{WW})$, we use the yields of $\mathrm{VG}$ for ILMS, and WW for massive stars (see Sect. 4). The calculated age-metallicity relation, metallicity distribution of $\mathrm{G}$ dwarfs and $[\mathrm{O} / \mathrm{Fe}]$ vs. $[\mathrm{Fe} / \mathrm{H}]$ relation can all fit the observations well (Figs. 1a,c,d). The predicted abundance 
Table 2. Selected parameters of stellar evolution and nucleosynthesis of massive stars.

\begin{tabular}{lllll}
\hline & WW & N97 & M92 & PCB \\
\hline upper mass & $40 M_{\odot}$ & $70 M_{\odot}$ & $120 M_{\odot}$ & $120 M_{\odot}$ \\
metallicity & $Z=1,0.1,0.01,10^{-4}, 0 Z_{\odot}$ & $Z_{\odot}$ & $Z=0.001,0.02$ & $Z=0.0004-0.05$ \\
stellar wind mass loss & No & No & Yes & Yes \\
${ }^{12} \mathrm{C}(\alpha, \gamma){ }^{16} \mathrm{O}$ rate & $1.7 \times \mathrm{CF} \simeq 0.74 \times \mathrm{CFHZ}$ & $\mathrm{CFHZ}$ & $\mathrm{CFHZ}$ & $\mathrm{CF}$ \\
\hline $\mathrm{CFHZ}=$ Caughlan et al. (1985). & & & & \\
$\mathrm{CF}=$ Caughlan \& Fowler (1988). & & & &
\end{tabular}

evolution of carbon is given in Fig. 1b; this shows that $[\mathrm{C} / \mathrm{Fe}]$ vs. $[\mathrm{Fe} / \mathrm{H}]$ is approximately constant in time. The predicted trend agrees with the observations, including even the increasing positive $[\mathrm{C} / \mathrm{Fe}]$ below $[\mathrm{Fe} / \mathrm{H}]<-1.5$. The reason of positive $[\mathrm{C} / \mathrm{Fe}]$ may be that for the massive stars, the $\mathrm{C}$ yields are high and the $\mathrm{Fe}$ yields are relative low. In medium metal-poor region, $[\mathrm{Fe} / \mathrm{H}]>-1.0$, $[\mathrm{C} / \mathrm{Fe}]$ slightly increases, showing the important $\mathrm{C}$ contribution from ILMS. Up to the late evolutionary stage of the Galaxy, the ratio of SN Ia rate to SN II rate increases, therefore the $[\mathrm{C} / \mathrm{Fe}]$ value decreases with increasing $[\mathrm{Fe} / \mathrm{H}]$ in the range of $[\mathrm{Fe} / \mathrm{H}]>-0.5$. The results show that the combined contribution of carbon by VG and WW can explain the observed $[\mathrm{C} / \mathrm{Fe}]$ vs. $[\mathrm{Fe} / \mathrm{H}]$ relation.

\section{2. $V G+N 97$}

Using the yields of N97 for massive stars and those of VG for ILMS (model VG+N97), our GCE results can fit the observational age-metallicity relation, metallicity distribution of $\mathrm{G}$ dwarfs and $[\mathrm{O} / \mathrm{Fe}]$ values (Figs. 2a,c,d). But the predicted $\mathrm{C}$ abundance is too low to fit the observations (Fig. 2b). Compared to $\mathrm{WW}$, the lower $\mathrm{C}$ yields calculated by N97 may be mainly caused by the choice of a higher rate of ${ }^{12} \mathrm{C}(\alpha, \gamma){ }^{16} \mathrm{O}$ reaction. Also, the authors of N97 themselves have compared the different nucleosynthesis results based on two different ${ }^{12} \mathrm{C}(\alpha, \gamma){ }^{16} \mathrm{O}$ reaction rates from Caughlan et al. (1985) (hereafter CFHZ) and Caughlan \& Fowler (1988) (hereafter CF) respectively. $\mathrm{CFHZ}$ gave a higher reaction rate than $\mathrm{CF}: \mathrm{CFHZ} \approx$ $2.3 \times \mathrm{CF}$; and $\mathrm{WW}$ adopted $1.7 \times \mathrm{CF} \approx 0.74 \times \mathrm{CFHZ}$ in their calculation, which is lower than in N97. So when $[\mathrm{O} / \mathrm{Fe}]$ can be matched, $[\mathrm{C} / \mathrm{Fe}]$ is lower than the observations. These results underline the importance of the ${ }^{12} \mathrm{C}(\alpha, \gamma){ }^{16} \mathrm{O}$ reaction rate to elemental nucleosynthesis.

Certainly, besides the ${ }^{12} \mathrm{C}(\alpha, \gamma){ }^{16} \mathrm{O}$ reaction rate, convective mechanism and explosion energy can affect the nucleosynthesis yields (N97).

\section{3. $V G+M 92$}

The results predicted by model VG+M92 can fit the observed age-metallicity relation, metallicity distribution of $\mathrm{G}$ dwarfs and $[\mathrm{O} / \mathrm{Fe}]$ vs. $[\mathrm{Fe} / \mathrm{H}]$ (Figs. 3a,c,d). There is a strong increase in the calculated $[\mathrm{C} / \mathrm{Fe}]$ for $[\mathrm{Fe} / \mathrm{H}]>-1.2$ (Fig. 3b). What is the main contributor? ILMS? Stellar winds of W-R stars? Or a combined contribution of the two? Let us try to identify the main source.
Figure 3e gives the result for only the yields of massive stars of M92. It shows the predicted $[\mathrm{C} / \mathrm{Fe}]$ increases with metallicity in metal-rich region. The calculated $[\mathrm{C} / \mathrm{Fe}]$ using the yields of ILMS (VG) and only massive stars with $M \leq 40 M_{\odot}(\mathrm{M} 92)$ is presented in Fig. 3f: here, the calculated $[\mathrm{C} / \mathrm{Fe}]$ is slightly lower than the observations in metal-rich region. The difference between Figs. 3e and $3 \mathrm{f}$ shows that the contribution to carbon from stellar wind of high metallicity W-R stars calculated by M92 is greater than the contribution from ILMS given by VG. The $M>40 M_{\odot}$ massive stars with higher metallicity, the W-R stars, can eject significant amounts of carbon, which causes the $[\mathrm{C} / \mathrm{Fe}]$ value to increase with increasing metallicity in the range of $[\mathrm{Fe} / \mathrm{H}]>-1.0$ in Fig. 3e.

\section{4. $R V+W W$}

Using results of RV as the yields of ILMS and WW for massive stars (model RV+WW), we calculated the corresponding age-metallicity relation, metallicity distribution of $\mathrm{G}$ dwarfs, $[\mathrm{O} / \mathrm{Fe}]$ vs. $[\mathrm{Fe} / \mathrm{H}]$ and $[\mathrm{C} / \mathrm{Fe}]$ vs. $[\mathrm{Fe} / \mathrm{H}]$ (Figs. 4a-d). When these three results match the observations, $[\mathrm{C} / \mathrm{Fe}]$ first increases slightly and then decreases, and is slightly higher than the prediction of model $\mathrm{VG}+\mathrm{WW}$ in metal-rich region. These results show that the RV calculation gives more carbon than does VG due to the use of different parameter values (see M2K, Liang \& Zhao 2001 for details).

\section{5. $R V+N 97$}

Figures 5a,c,d show that the calculated results using model $\mathrm{RV}+\mathrm{N} 97$ can fit the observations, i.e., the age-metallicity relation, the metallicity distribution of $\mathrm{G}$ dwarfs and the $[\mathrm{O} / \mathrm{Fe}]$ vs. $[\mathrm{Fe} / \mathrm{H}]$ relation. The calculated $[\mathrm{C} / \mathrm{Fe}]$ vs. $[\mathrm{Fe} / \mathrm{H}]$ then shows that more carbon is given by RV than $\mathrm{VG}$, and this increases the predicted $[\mathrm{C} / \mathrm{Fe}]$ in metal-rich region (Fig. 5b). Because N97 gives low carbon yields for the reason of adopting a low ${ }^{12} \mathrm{C}(\alpha, \gamma){ }^{16} \mathrm{O}$ reaction rate, $[\mathrm{C} / \mathrm{Fe}]$ falls below the observations in the whole metallicity range (Fig. $5 \mathrm{~b}$ ).

\section{6. $R V+M 92$}

Figures $6 \mathrm{a}-\mathrm{d}$ display the results based on the yields of RV for ILMS and of M92 for massive stars (model RV+M92). Figs. 6a,c,d show the calculations can fit the corresponding observations, while Fig. $6 \mathrm{~b}$ shows that $[\mathrm{C} / \mathrm{Fe}]$ increases 

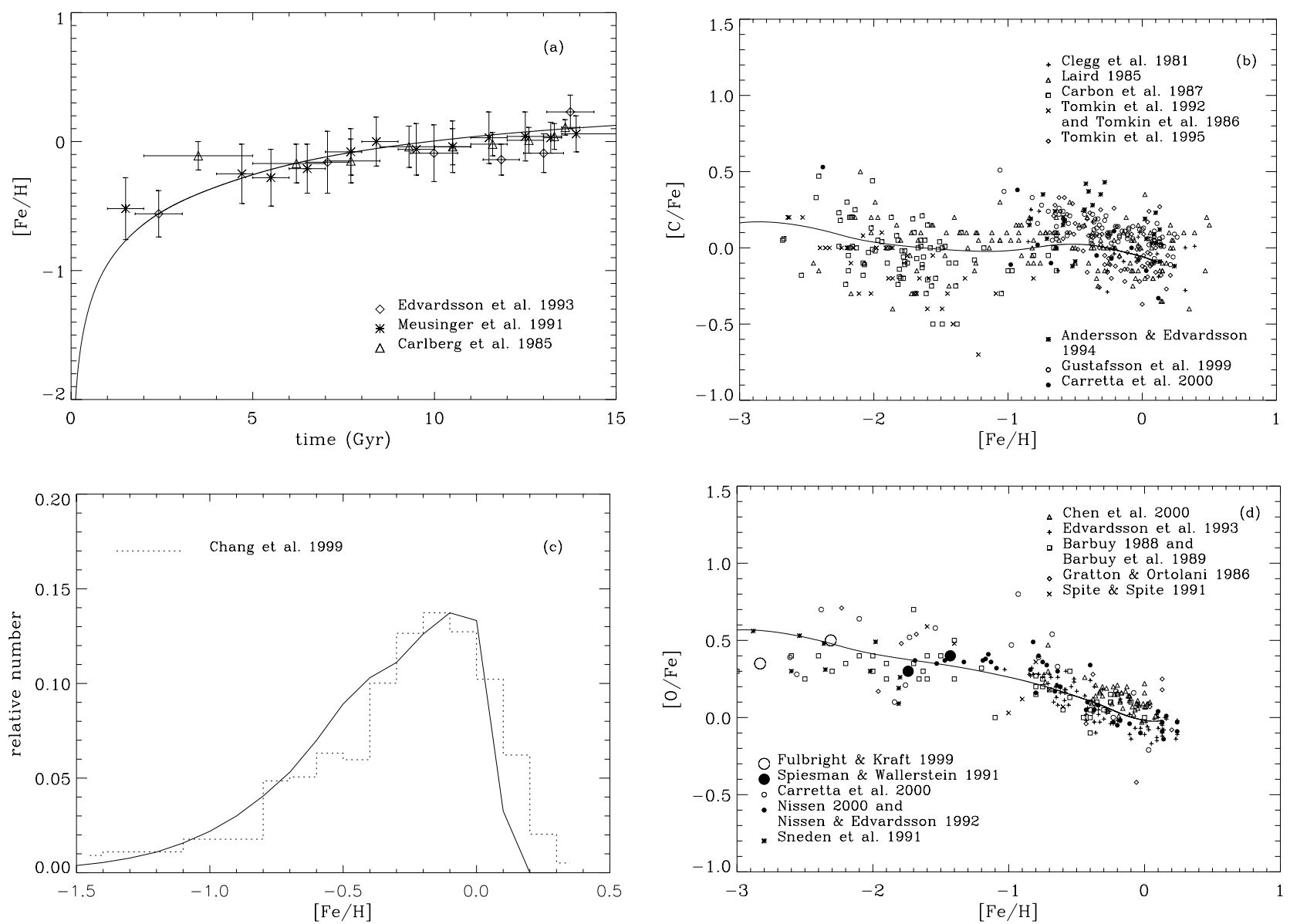

Fig. 1. Results using the nucleosynthesis yields of VG for ILMS and WW for massive stars (the solid lines) (model VG+WW). a) The age-metallicity relation in the solar neighborhood; b) The $[\mathrm{C} / \mathrm{Fe}]$ vs. $[\mathrm{Fe} / \mathrm{H}]$ relation; c) The metallicity distribution of $\mathrm{G}$ dwarfs in the solar neighborhood; $\mathbf{d})$ The $[\mathrm{O} / \mathrm{Fe}]$ vs. $[\mathrm{Fe} / \mathrm{H}]$ relation. The corresponding observations are shown in the figures.

with metallicity in metal-rich region (Fig. 6b). There are no apparent difference here between this model and the model VG+M92 (Fig. 3b); this can be understood because the $\mathrm{C}$ contribution from $\mathrm{W}-\mathrm{R}$ stars given by M92 is higher than that from ILMS given by RV or VG.

Figure $6 \mathrm{e}$ shows the $[\mathrm{C} / \mathrm{Fe}]$ vs. $[\mathrm{Fe} / \mathrm{H}]$ relation based only on the yields of massive stars from M92; with no contribution from ILMS, this is still higher than the observations in metal-rich region. Figure $6 \mathrm{f}$ exhibits the predicted $[\mathrm{C} / \mathrm{Fe}]$ using the yields of RV for ILMS and those of M92 for massive stars with $M \leq 40 M_{\odot}$; it fits the observations, and shows a slightly higher $[\mathrm{C} / \mathrm{Fe}]$ than is given by model VG+M92 in metal-rich region (Fig. 3b). This result shows that RV gives higher $\mathrm{C}$ yields than does VG with a different choice of the parameters.

\section{7. $M B C+P C B$}

It is proper to combine the yields of $\mathrm{MBC}$ and $\mathrm{PCB}$ together since they used the same parameters (Padova group). They adopted a larger convective overshooting, so that stars with $M>6 M_{\odot}$ rather than the standard $>8 M_{\odot}$ would end their lives through SN II explosions. Both the age-metallicity relation and metallicity distribution of $\mathrm{G}$ dwarfs can fit the observations well (Figs. 7a,c). The $\mathrm{O}$ abundance is slightly lower than the observations (Fig. 7d), which may be caused by the lower ${ }^{12} \mathrm{C}(\alpha, \gamma){ }^{16} \mathrm{O}$ reaction rate $(\mathrm{CF})$ used by the Padova group. The predicted $[\mathrm{C} / \mathrm{Fe}]$ vs. $[\mathrm{Fe} / \mathrm{H}]$ relation is given in Fig. $7 \mathrm{~b}$, which shows a strong increase of $[\mathrm{C} / \mathrm{Fe}]$ with increasing metallicity from $[\mathrm{Fe} / \mathrm{H}] \approx-0.7$ on.

Figure $7 \mathrm{e}$ displays the $[\mathrm{C} / \mathrm{Fe}]$ based only on the contribution of carbon from massive stars; it shows that $\mathrm{W}$ $\mathrm{R}$ stars with a high metallicity $(Z=0.008,0.02)$ can eject significant amounts of carbon into the ISM, much more than the lower metallicity stars. Thus, $[\mathrm{C} / \mathrm{Fe}]$ increases steeply starting from $[\mathrm{Fe} / \mathrm{H}] \approx-0.7$. The subsequent decrease from $[\mathrm{Fe} / \mathrm{H}] \approx-0.3$ on is the result of $\mathrm{Fe}$ contribution from SN Ia explosions.

Figure $7 \mathrm{f}$ shows the case of only ILMS and the $M \leq$ $40 M_{\odot}$ part of massive stars; there is basic fit to the observations. Comparing Figs. 7b, 7e and 7f, we find the main effect of the $\mathrm{C}$ yields from metal-rich $\mathrm{W}-\mathrm{R}$ stars is the 

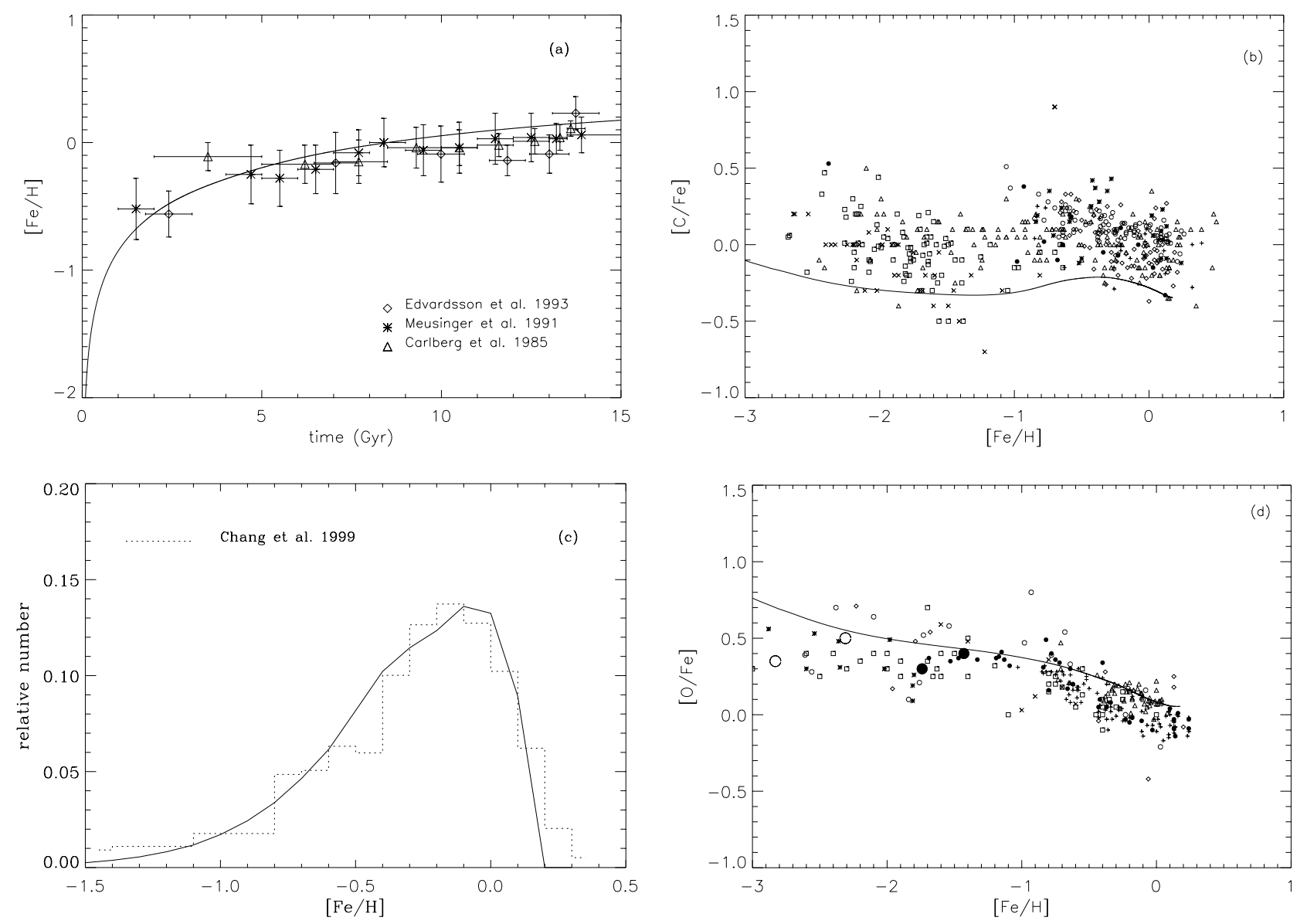

Fig. 2. Same as Fig. 1, but with the nucleosynthesis yields of VG for ILMS and N97 for massive stars (model VG+N97).

obvious increase of $[\mathrm{C} / \mathrm{Fe}]$ in metal-rich region shown in Fig. $7 \mathrm{~b} ;-$ an increase to above the observations.

\section{8. $M 2 K+P C B$}

We use the nucleosynthesis yields of M2K in our GCE calculations because this new set of yields include the results of ILMS with the low metallicity value $Z=0.004$, which may contain significant information on the ILMS during the early Galactic stage.

We combine $\mathrm{M} 2 \mathrm{~K}$ and $\mathrm{PCB}$ in our model $\mathrm{M} 2 \mathrm{~K}+\mathrm{PCB}$. The calculated age-metallicity relation, metallicity distribution of $\mathrm{G}$ dwarfs and $[\mathrm{O} / \mathrm{Fe}]$ vs. $[\mathrm{Fe} / \mathrm{H}]$ relation again match the observations, and are similar to the results of model $\mathrm{MBC}+\mathrm{PCB}$, and so are not shown here. The predicted evolution of $[\mathrm{C} / \mathrm{Fe}]$ with metallicity is given in Fig. 8a, which shows a strong increase with increasing metallicity from $[\mathrm{Fe} / \mathrm{H}] \approx-1.5 \mathrm{on}$. In order to understand this increase, we re-calculate the $[\mathrm{C} / \mathrm{Fe}]$ vs. $[\mathrm{Fe} / \mathrm{H}]$ relation using only the contribution of massive stars (Fig. 8c), and only the yields of ILMS and $M \leq 40 M_{\odot}$ massive stars (Fig. 8d) respectively. Comparing Figs. 8c and 7e, and Figs. $8 \mathrm{~d}$ and $7 \mathrm{f}$, we guess that maybe it is the ILMS with $Z=0.004$ that produce a significant amount of carbon, which lead to the strong increase of $[\mathrm{C} / \mathrm{Fe}]$ displayed in
Fig. 8a. Figure 8e confirms our guess, in which the solid line represents the calculated $[\mathrm{C} / \mathrm{Fe}]$ vs. $[\mathrm{Fe} / \mathrm{H}]$ relation using only the yields of $Z=0.008,0.02$ (the same metallicities as in $\mathrm{MBC}$ ) of $\mathrm{M} 2 \mathrm{~K}$ for ILMS and $\mathrm{PBC}$ for massive stars, and the dashed line represents the results of model MBC+PCB (the same line as in Fig. 7b).

The solid and dashed lines in Fig. 8f represent the corresponding results for nitrogen. Figs. 8e, $\mathrm{f}$ show that the nitrogen yields of M2K with $Z=0.008,0.02$ are very similar to the corresponding results of $\mathrm{MBC}$, though $\mathrm{M} 2 \mathrm{~K}$ gave more carbon and nitrogen according to the updated parameters, resulting in the slightly higher $[\mathrm{C} / \mathrm{Fe}]$ and $[\mathrm{N} / \mathrm{Fe}]$ values in metal-rich region. Figure $8 \mathrm{~b}$ illustrates the $[\mathrm{N} / \mathrm{Fe}]$ vs. $[\mathrm{Fe} / \mathrm{H}]$ relation using the yields of $\mathrm{M} 2 \mathrm{~K}$ for ILMS; the relation is higher than that shown in Fig. 8f.

These results show that ILMS with low metallicity $Z=$ 0.004 contribute very important amounts of $\mathrm{C}$ and $\mathrm{N}$ to the ISM (also see Fig. 1 of M2K). The lower metallicity ILMS have higher $\mathrm{C}$ and $\mathrm{N}$ abundances because for them the HBB process is more efficient, and mass loss is less so. The lower mass loss rates correspond to longer TP-AGB lifetimes, hence a greater number of the third dredge-up events and a longer duration of the HBB process. The up-to-date nucleosynthesis calculations (VG) only go as low as $Z=0.001$. Calculations of ILMS of even lower metallicities are needed; these may revise the trends of 

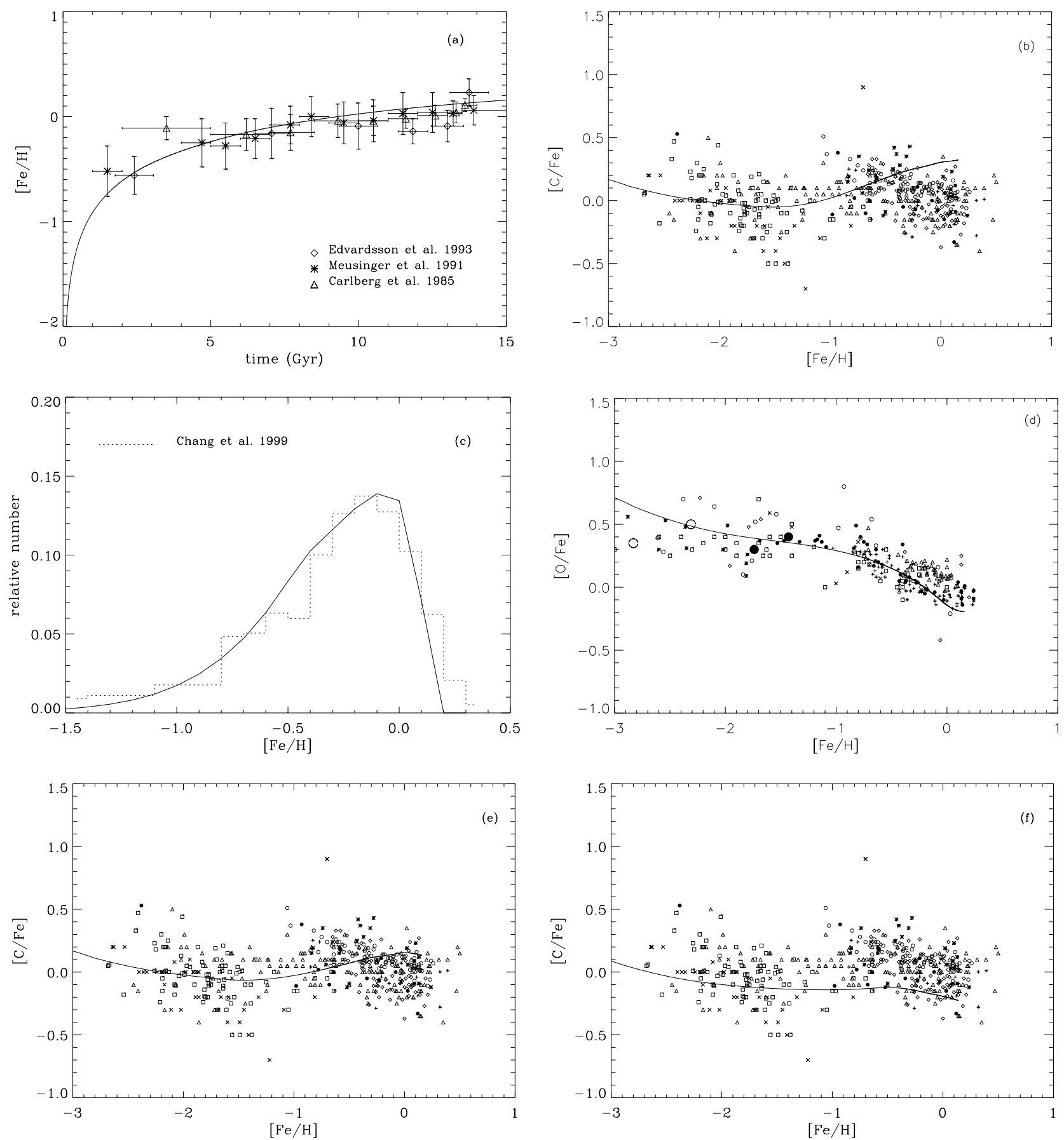

Fig. 3. a-d) are same as in Fig. 1, but with the nucleosynthesis yields of VG for ILMS and M92 for massive stars (model $\mathrm{VG}+\mathrm{M} 92)$. e) The calculated $[\mathrm{C} / \mathrm{Fe}]$ vs. $[\mathrm{Fe} / \mathrm{H}]$ only considering the $\mathrm{C}$ yields of massive stars of M92; f) The calculated $[\mathrm{C} / \mathrm{Fe}]$ vs. $[\mathrm{Fe} / \mathrm{H}]$ considering $\mathrm{C}$ yields of $\mathrm{VG}$ for ILMS and $\mathrm{C}$ yields of only $M \leq 40 M_{\odot}$ stars from M92 for massive stars.

elemental abundance evolution, and provide different hints to the early GCE history.

Though the nucleosynthesis results with $\alpha=1.68$ and 2.00 given by $\mathrm{M} 2 \mathrm{~K}$ are different (more nitrogen is produced by intermediate mass stars with $\alpha=2.00$ than with $\alpha=1.68$ ), we find no obvious differences between these two sets of results. So we only give the GCE results based on the yields with $\alpha=2.00$ in this paper.

In summary, we give and analyze the calculated abundance evolution of carbon using 8 recently published sets of nucleosynthesis yields. The parameters of the 8 models are listed in Table 3 . Here are some of the obvious implications of the parameters. The higher the $\zeta$ value, the more contribution comes from massive stars, and then the predicted $\mathrm{O}$ abundance will be higher, especially in low metallicity region. A higher $\beta$ value means a higher SN Ia rate, hence a greater amount of Fe element from SN Ia explosion. A higher $\nu$ value represents a higher SFR, more stars form, leading to an increase in the $\mathrm{O}$ abundance and $[\mathrm{Fe} / \mathrm{H}]$, and the effect is more obvious in metal-poor 

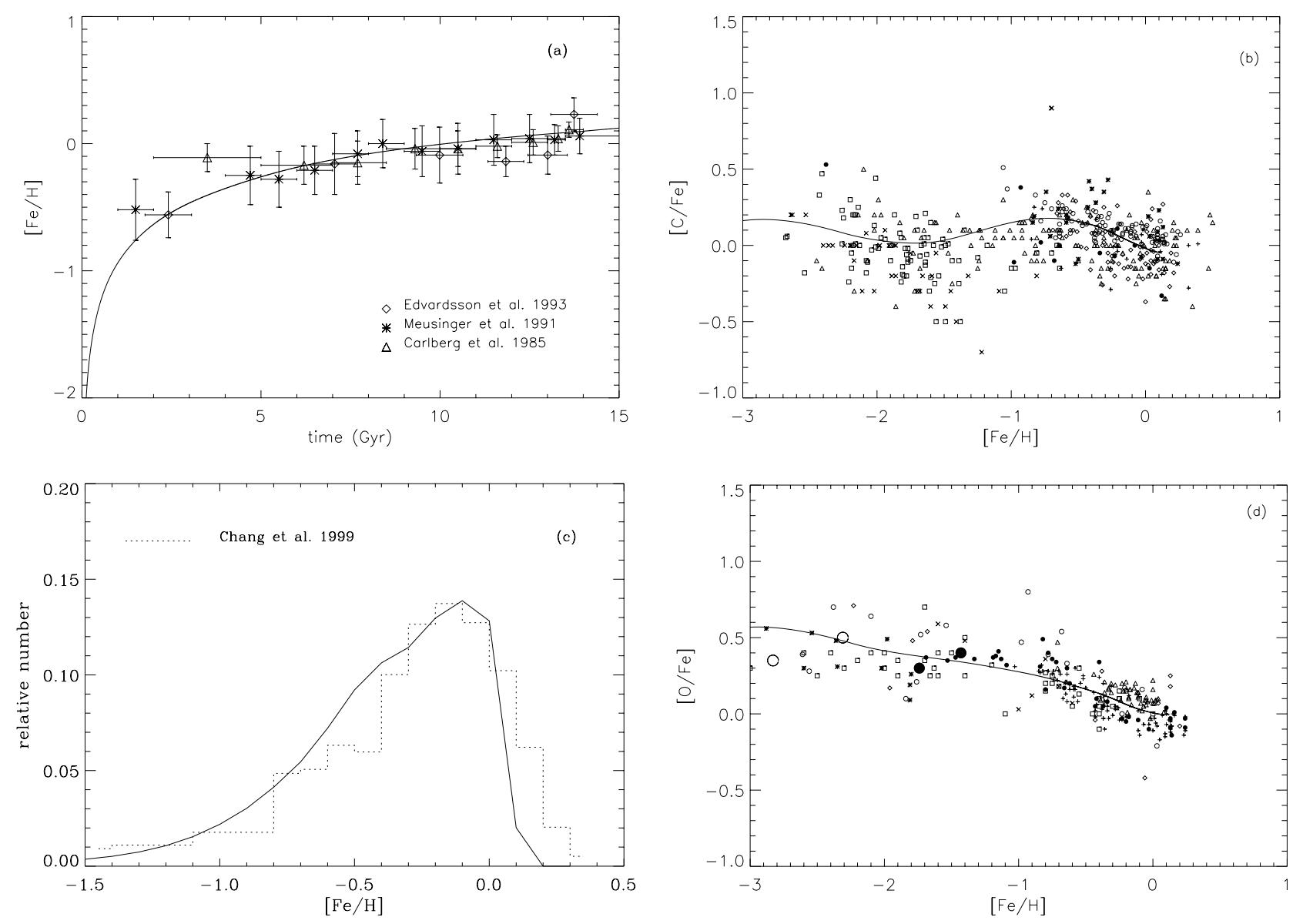

Fig. 4. Same as Fig. 1, but with the nucleosynthesis yields of RV for ILMS and WW for massive stars (model RV+WW).

Table 3. Input parameters of our 8 models.

\begin{tabular}{llll|llll}
\hline model & $\zeta$ & $\beta$ & $\nu$ & model & $\zeta$ & $\beta$ & $\nu$ \\
\hline VG+WW & 0.51 & 0.037 & 1.0 & RV+WW & 0.54 & 0.033 & 1.0 \\
VG+N97 & 0.40 & 0.045 & 1.0 & RV+N97 & 0.40 & 0.040 & 1.0 \\
VG+M92 & 0.40 & 0.040 & 0.7 & RV+M92 & 0.40 & 0.035 & 0.7 \\
MBC+PCB & 0.37 & 0.045 & 0.5 & M2K+PCB & 0.37 & 0.045 & 0.5 \\
\hline
\end{tabular}

region. At the same time, a higher $\nu$ will lead to a higher peak in the metallicity distribution of $\mathrm{G}$ dwarfs.

\subsection{The ${ }^{12} \mathrm{C}(\alpha, \gamma){ }^{16} \mathrm{O}$ reaction rate}

The ${ }^{12} \mathrm{C}(\alpha, \gamma){ }^{16} \mathrm{O}$ reaction rate is very important to stellar nucleosynthesis. It determines the $\mathrm{C}$ and $\mathrm{O}$ abundances, hence the abundances of the heavier elements. Generally, in nucleosynthesis calculations, the ${ }^{12} \mathrm{C}(\alpha, \gamma){ }^{16} \mathrm{O}$ reaction rates given by $\mathrm{CFHZ}$ and $\mathrm{CF}$ are widely used. $\mathrm{CFHZ}$ gives higher values than does $\mathrm{CF}$ : $\mathrm{CFHZ} \approx 2.3 \times \mathrm{CF}$. And the higher reaction rate leads to a lower $\mathrm{C}$ abundance. N97 exhibited this result in their Fig. 7. They suggested that the actual reaction rate was between $\mathrm{CFHZ}$ and $\mathrm{CF}$. WW adopted $1.7 \times \mathrm{CF}(\approx 0.74 \times \mathrm{CFHZ})$ to be the actual reaction rate in their nucleosynthesis calculations. They gave higher $\mathrm{C}$ yields than did N97.
For the higher massive stars, stellar wind mass loss strongly affects the $\mathrm{C}$ yields, so the effect caused by the ${ }^{12} \mathrm{C}(\alpha, \gamma){ }^{16} \mathrm{O}$ reaction rate is obscured. The lower reaction rate of $\mathrm{CF}$ has led to the lower $\mathrm{O}$ yields of $\mathrm{PCB}$.

\subsection{Understanding the $W-R$ stars}

Leitherer (1995) suggested that the most massive stars known in the universe have masses around $100 M_{\odot}$ and lifetimes of a few Myrs. They are rare: in the solar neighborhood only about one such massive star is counted per $10^{5}$ to $10^{6}$ solar-type stars. There are three key phases during the evolution of a massive star: O star, luminous blue variable star and Wolf-Rayet star. The mass loss of a massive star can be calculated from UV wind lines, $\mathrm{H}_{\alpha}$ or radio fluxes. The UV $\mathrm{P}$ Cygni profile, ubiquitous in O-type stars, provides a direct indication of stellar wind; $\mathrm{H}_{\alpha}$ has been recognized as the prime source of mass-loss 

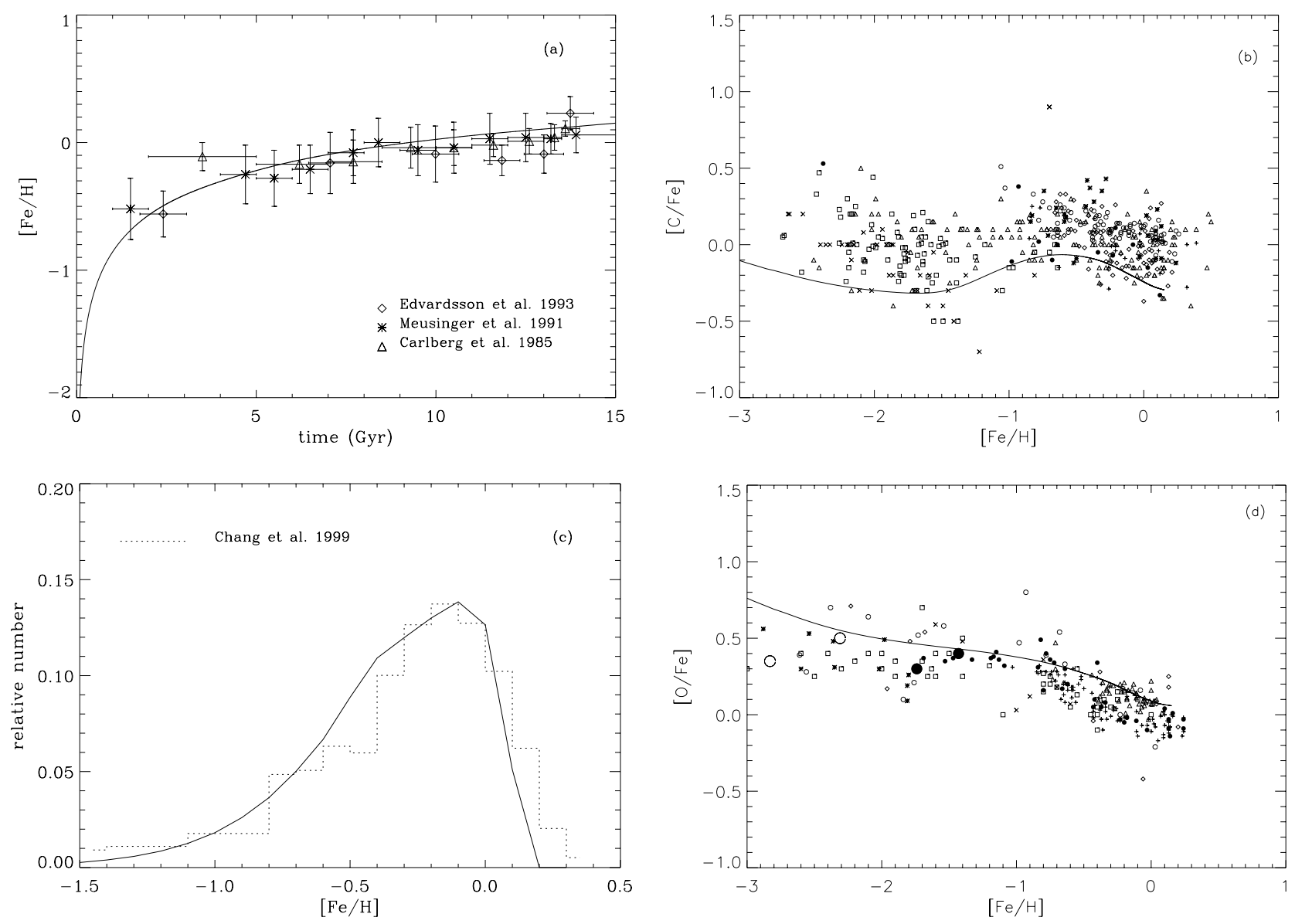

Fig. 5. Same as Fig. 1, but with the nucleosynthesis yields of RV for ILMS and N97 for massive stars (model RV+N97).

in early-type stars; and winds in hot stars can be readily observed at IR-mm-radio wavelengths via the free-free "thermal" excess caused by the stellar wind. The mass loss rate scales as $Z^{0.5}$ for $\mathrm{O}$ stars and early B stars, as $Z^{0.8}$ for mid-B supergiants, and as $Z^{1.7}$ for A supergiants (Kudritzki \& Puls 2000; Crowther 2000a).

W-R stars are easily detectable due to their intrinsic brightness and the profound effects on the interstellar environment. They eject a large amount of material into the ISM via the radiative-driven stellar wind. The nucleosynthesis calculations show that the ejected carbon by massive stars are very important to the enrichment of the ISM. In general, the mass loss rate of massive stars adopted in the stellar evolution calculations are $\dot{M} \sim M^{2.5}$ and $\dot{M} \sim Z^{0.5}$ (M92; PCB). However, according to recent reviews, our knowledge of the mass loss of massive stars is not very clear.

Meynet \& Maeder (2000) calculated the evolution of stars with rotation, and suggested that rotation can increase mass loss, also can bring significant surface Heand $\mathrm{N}$-enhancements; the enhancements are the greater for the higher the mass and the rotation. In his review, Crowther (2000a) found that the recent mass-loss rates for Galactic W-R stars indicated a downward revision of 2-4 relative to previous calibrations (e.g. Langer 1989:
$\dot{M} \sim M^{2.5}$ ) due to clumping (e.g. Schmutz 1997). Indeed, metallicity affects the yields of carbon. When mass loss gets very efficient, ${ }^{12} \mathrm{C}$ will increase because He-burning products can be revealed on the surface. If mass loss is extreme, anyway, ${ }^{12} \mathrm{C}$ and ${ }^{16} \mathrm{O}$ yields may even decrease because most of the mass is rapidly lost in the wind in the form of ${ }^{4} \mathrm{He}$. But at present, the question of a $Z$-dependent mass loss in W-R stars remains open. Crowther (2000a) suggested that there is a clear distinction among spectral subtypes in galaxies with different metallicities, which is qualitatively explained by evolutionary and spectroscopic models (Crowther 2000b). WN stars in LMC show negligible spectroscopic difference from their Galactic counterparts. And the situation is less clear for the SMC, since most W-R stars are complicated by binarity (Crowther 2000a).

\subsection{Contributions by stars of different masses}

Comparing our calculated results using different nucleosynthesis yields (Sects. 5.1-5.8), considering the uncertainty of stellar wind mass loss of massive stars (see Sect. 5.10), and assuming that the actual ${ }^{12} \mathrm{C}(\alpha, \gamma){ }^{16} \mathrm{O}$ reaction rate to be between the values of $\mathrm{CFHZ}$ and $\mathrm{CF}$, we 

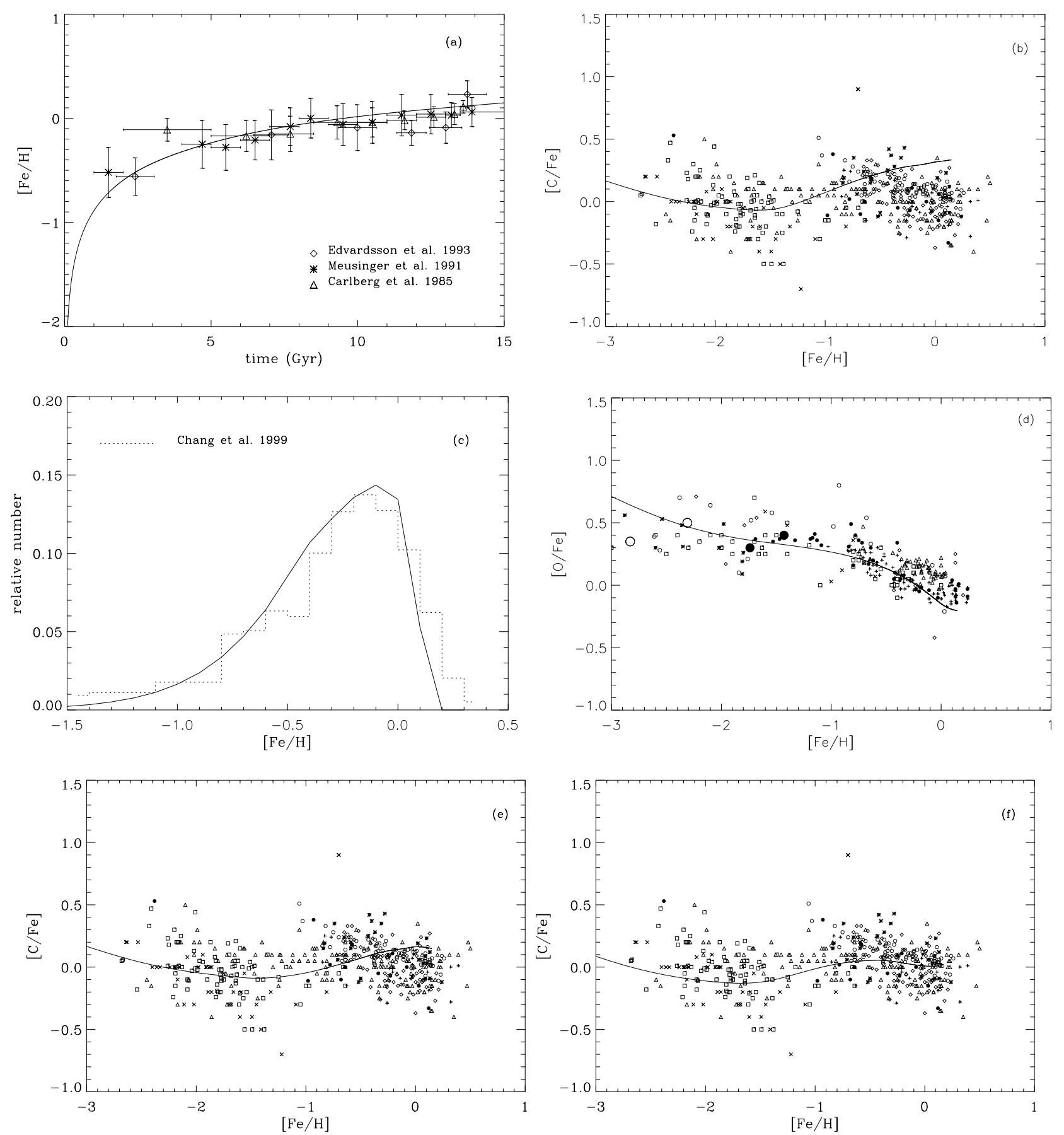

Fig. 6. Same as Fig. 3, but with nucleosynthesis yields of RV for ILMS and M92 for massive stars (model RV+M92).

choose to use the results of model $\mathrm{VG}+\mathrm{WW}$ in an analysis of the contributions of carbon by stars of different masses.

Here, the reason of choosing VG rather than RV for ILMS is because of suggestions that the yields of RV are not successful in predicting the observed $\mathrm{C} / \mathrm{O}$ and $\mathrm{N} / \mathrm{O}$ ratios in stars and planetary nebulae, or in reproducing the carbon stars of relatively low luminosities in the Magellanic Clouds (Gustafsson et al. 1999).

Figure 9 shows the fraction of carbon contributed by massive stars based on the WW yields for massive stars and the VG yields for ILMS, as a function of the Galactic age. In the early Galactic stage, almost all the carbon is produced by massive stars. As the age advances, ILMS contribute more and more, and an overtake the massive stars from about 1.65 Gyr on. At the present epoch, many low mass stars have also evolved and ejected matter into the ISM, and the relative contribution by the ILMS is further increased.

\section{Nitrogen abundance}

The element nitrogen includes a primary and a secondary component. If nitrogen production depends on the initial $\mathrm{CNO}$ abundance, the $[\mathrm{N} / \mathrm{Fe}]$ would be dependent on 

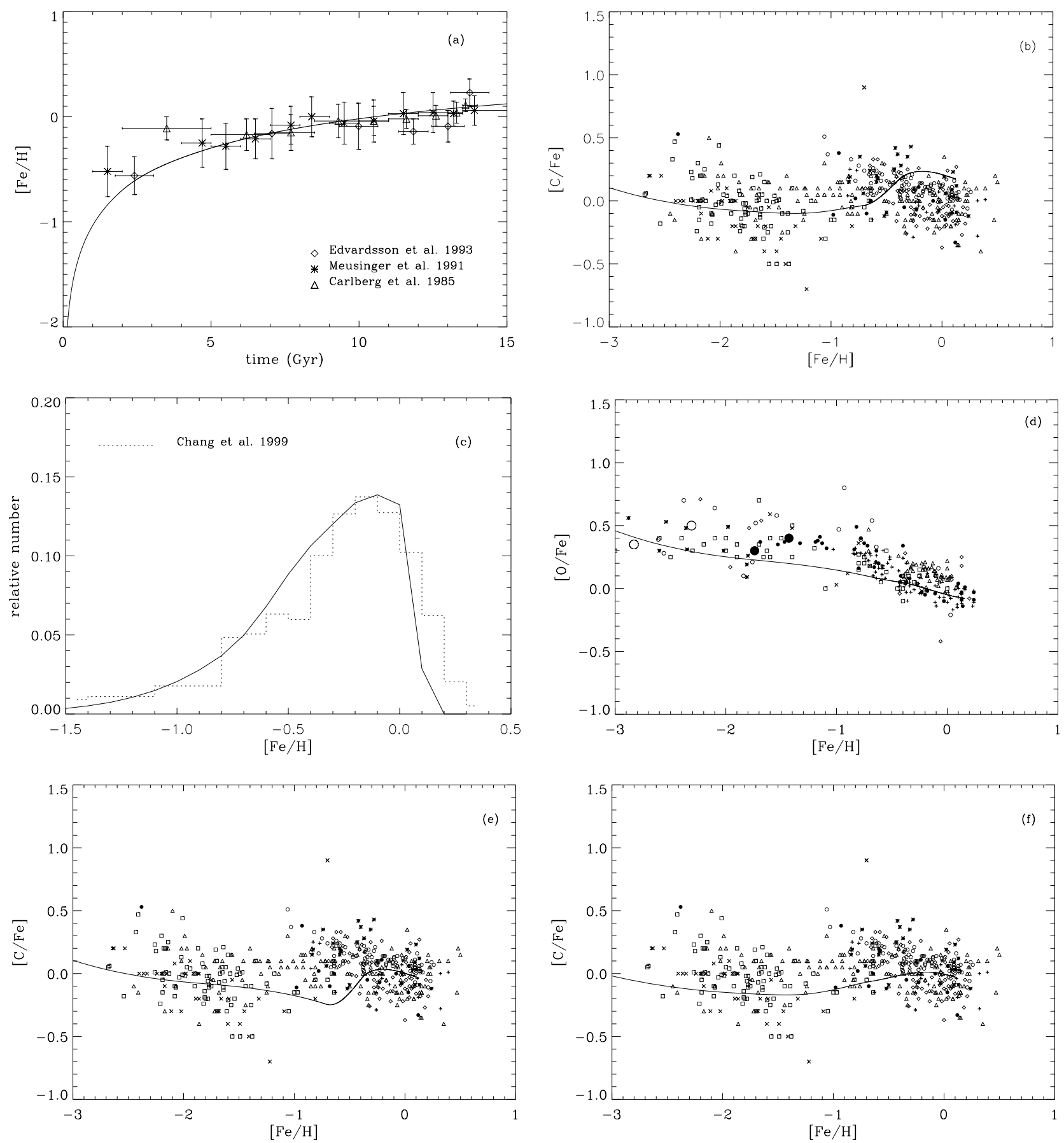

Fig. 7. Same as Fig. 3, but with nucleosynthesis yields of MBC for ILMS and PCB for massive stars (model MBC+PCB).

$[\mathrm{Fe} / \mathrm{H}]$, and this part of nitrogen is secondary; however, if nitrogen production is chiefly primary, then $[\mathrm{N} / \mathrm{Fe}]$ would be independent of the value of $[\mathrm{Fe} / \mathrm{H}]$.

Laird (1985) and Carbon et al. (1987) found the observed $[\mathrm{N} / \mathrm{Fe}]$ to be constant over the entire metallicity range, although the precise level is uncertain. So, it seems that the observations require a primary nitrogen source from massive stars. However, if the observed constant nitrogen in low metallicity region is correct, then the present yields by massive stars cannot supply the needed primary nitrogen source (Timmes et al. 1995; Chiappini et al. 1997). Our results, based on the recently published nucleosynthesis yields of massive stars, also point to an absence of a primary nitrogen source. Figures $10 \mathrm{a}-\mathrm{c}$ show the calculated results of $[\mathrm{N} / \mathrm{Fe}]$ vs. $[\mathrm{Fe} / \mathrm{H}]$ using our GCE models VG+WW, VG+N97 and VG+M92 respectively. Those models with the RV yields of ILMS give similar trends to these with the VG yields; so we do not show them here. The $[\mathrm{N} / \mathrm{Fe}]$ values of model $\mathrm{MBC}+\mathrm{PCB}$ and $\mathrm{M} 2 \mathrm{~K}+\mathrm{PCB}$ will be given in Sect. 5.8.

In these figures, the solid lines represent the results with the $\mathrm{N}$ yields of both ILMS and massive stars, and the dashed lines represent the results with only the contribution from massive stars. These results show that ILMS 

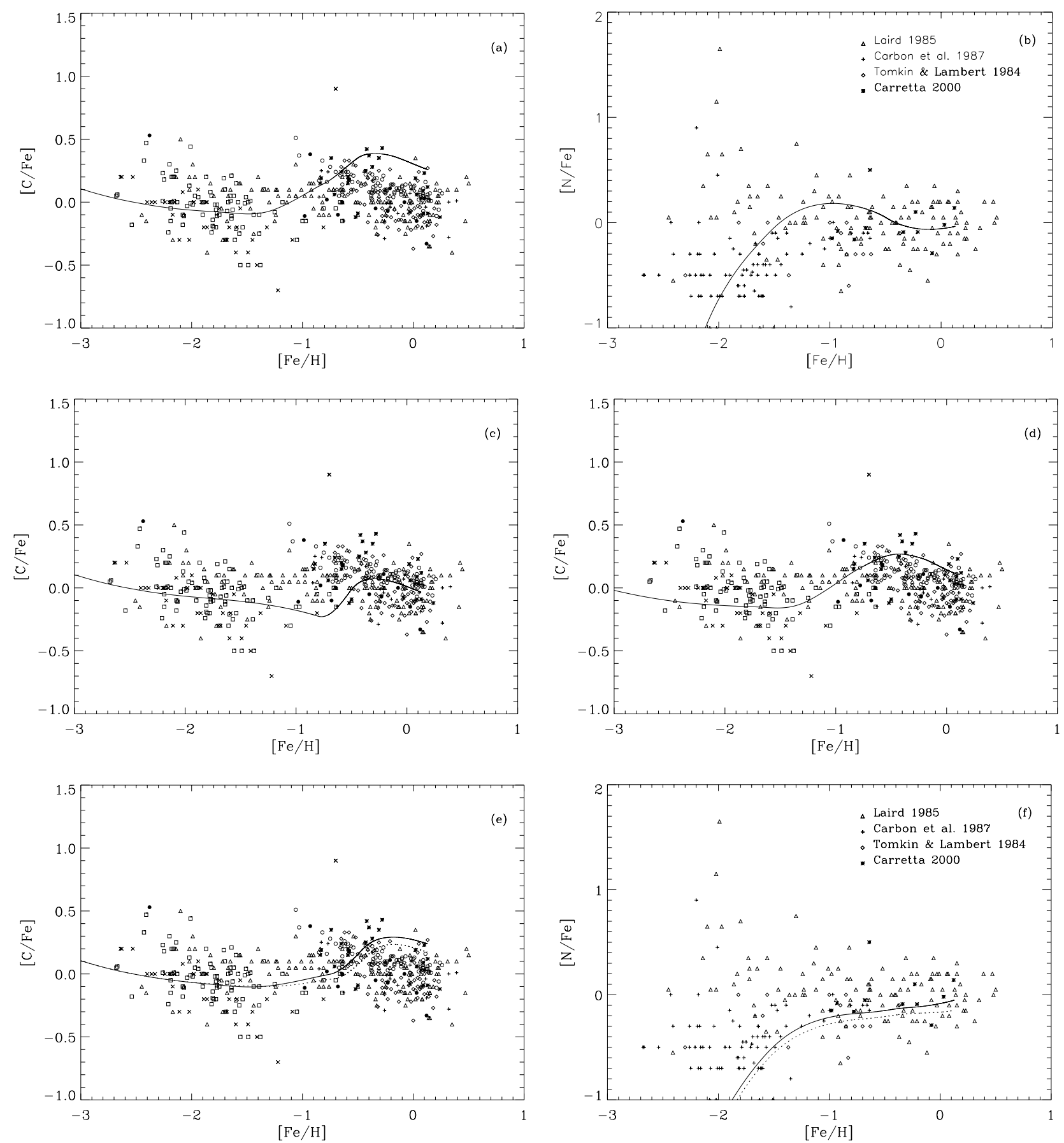

Fig. 8. a) The calculated $[\mathrm{C} / \mathrm{Fe}]$ vs. $[\mathrm{Fe} / \mathrm{H}]$ using nucleosynthesis yields of M2K for ILMS and PCB for massive stars (model $\mathrm{M} 2 \mathrm{~K}+\mathrm{PCB})$; b) Same as a) but for nitrogen; c) The calculated $[\mathrm{C} / \mathrm{Fe}]$ vs. $[\mathrm{Fe} / \mathrm{H}]$ considering only yields of massive stars from PCB without ILMS; d) The calculated $[\mathrm{C} / \mathrm{Fe}]$ vs. $[\mathrm{Fe} / \mathrm{H}]$ considering yields of M2K for ILMS and only $M \leq 40 M_{\odot}$ massive stars from PCB; e) The solid line represents the $[\mathrm{C} / \mathrm{Fe}]$ vs. $[\mathrm{Fe} / \mathrm{H}]$ relation considering the yields of $\mathrm{PCB}$ for massive stars and M2K only with $Z=0.008$ and $Z=0.02$ metallicities for ILMS, and the dashed line represents the same result as in Fig. $7 \mathrm{~b}$; f) Same as e) but for nitrogen.

are the dominant donor of nitrogen, while massive stars have provided only a small part to the ISM during the whole Galactic age up to the present. However, in the range of $-3<[\mathrm{Fe} / \mathrm{H}]<-2,[\mathrm{~N} / \mathrm{Fe}]$ increases steeply (solid lines in Figs. 10a, c), due to intermediate mass stars as source of secondary nitrogen. As metallicity increases up to $[\mathrm{Fe} / \mathrm{H}] \approx-1.0,[\mathrm{~N} / \mathrm{Fe}]$ increases gradually, - typical behaviour of secondary nitrogen. As $[\mathrm{Fe} / \mathrm{H}]$ increases further,
$[\mathrm{N} / \mathrm{Fe}]$ approaches the solar value, which shows that the primary nitrogen from LIMS plays an important role in the production of nitrogen since that time.

From another aspect, comparison between the results shown in Figs. 10a and 10c confirms further that the ILMS are the main source of nitrogen. These two figures are based the models VG+WW and VG+M92 which use different $\mathrm{N}$ yields from massive stars, yet the results are very 


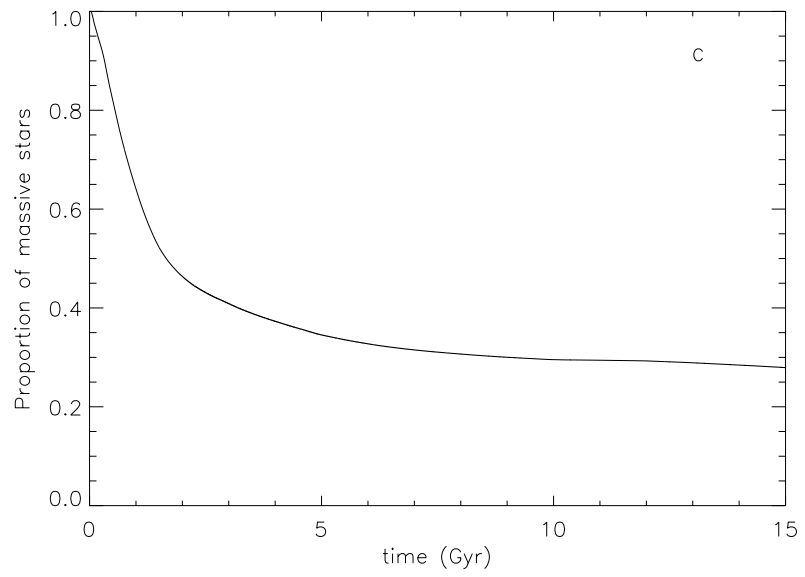

Fig. 9. The relative contribution of massive stars to carbon production at different Galactic ages using the results of WW for nucleosynthesis yields of massive stars and VG for ILMS.

similar, so we infer that it is the $\mathrm{N}$ yields of ILMS that provide the bulk of nitrogen to the ISM. In addition, we should note that M92 gives only the $\mathrm{N}$ yields of stellar wind and does not include contribution from SN II explosions, so the dashed line is higher in Fig. 10a than in Fig. 10c. Again, the predicted $\mathrm{N}$ abundance evolution by the models $\mathrm{MBC}+\mathrm{PCB}$ and $\mathrm{M} 2 \mathrm{~K}+\mathrm{PCB}$ also support the statement that ILMS are the dominant $\mathrm{N}$ contributor (see Figs. 8b, f). The model $\mathrm{VG}+\mathrm{N} 97$ predicts high $[\mathrm{N} / \mathrm{Fe}]$ in early Galactic stage, though not as high as the solar abundance. A possible reason is that N97 only gives the yields of solar metallicity massive stars, which eject more nitrogen than do low metallicity stars. With increasing metallicity, Fe from SN Ia explosions plays an increasingly more important role in the enrichment of the ISM, and this leads to a $[\mathrm{N} / \mathrm{Fe}]$ decrease with the Galactic evolution (dashed line in Fig. 10b).

In general, the relative weight of the secondary and primary components on the theoretical yield of ${ }^{14} \mathrm{~N}$ of ILMS depends on the interplay between the secondary enrichment caused by the first and second dredge-up episodes, and the primary contribution given by the CNO-cycle during the envelope burning. ${ }^{13} \mathrm{C}$ and ${ }^{14} \mathrm{~N}$ are regarded as secondary elements in the sense that they are formed from ${ }^{12} \mathrm{C}$ and ${ }^{16} \mathrm{O}$ originally present in the star at the time of its formation. Typical in this sense is the secondary production of ${ }^{13} \mathrm{C}$ and ${ }^{14} \mathrm{~N}$ during the first dredge-up, and the secondary production of ${ }^{14} \mathrm{~N}$ during the second dredgeup. However, when the third dredge-up process operates in conjunction with the HBB process, primary ${ }^{13} \mathrm{C}$ and ${ }^{14} \mathrm{~N}$ are produced. Meanwhile the primary ${ }^{12} \mathrm{C}$, dredged up after each He-shell flash is later converted in part into primary ${ }^{13} \mathrm{C}$ and ${ }^{14} \mathrm{~N}$ by the burning at the base of the convective envelope during the interpulse phase.

Is it sure that the observed $[\mathrm{N} / \mathrm{Fe}]$ is near 0.0 in metal-poor region? Carbon et al. (1987) found two stars with high $\mathrm{N}$ abundances, HD 74000 with $[\mathrm{N} / \mathrm{Fe}] \sim+0.9$, HD 25329 with $[\mathrm{N} / \mathrm{Fe}] \sim+0.45$. Laird (1985) obtained high $\mathrm{N}$ abundances for four stars, HD 74000 with
$[\mathrm{N} / \mathrm{Fe}] \sim+1.15$ and $[\mathrm{Fe} / \mathrm{H}]=-2.02$, HD 97916 with $[\mathrm{N} / \mathrm{Fe}] \sim+0.75$ and $[\mathrm{Fe} / \mathrm{H}]=-1.30, \mathrm{HD} 160617$ with $[\mathrm{N} / \mathrm{Fe}] \sim+1.65$ and $[\mathrm{Fe} / \mathrm{H}]=-1.99$, HD 166913 with $[\mathrm{N} / \mathrm{Fe}] \sim+0.70$ and $[\mathrm{Fe} / \mathrm{H}]=-1.80$. The possible reasons for the very high $\mathrm{N}$ abundance given by the authors are: (1) internal mixing; (2) mass transfer in a binary system; (3) primordial N enhancement. Beveridge \& Sneden (1994) reanalyzed the chemical abundances of two N-rich dwarf stars, namely HD 74000 and HD 25329. Their results showed that all the very heavy elements synthesized through neutron-capture s-process were enhanced in these two stars. It is likely that the enrichment of $\mathrm{N}$ and the very heavy elements originated in material dredged up from the helium burning shell of former AGB stars. Also, Carbon et al. (1987) pointed out that, omitting the N-rich objects, the observed $[\mathrm{N} / \mathrm{Fe}]$ vs. $[\mathrm{Fe} / \mathrm{H}]$ plot showed a negative correlation with $[\mathrm{Fe} / \mathrm{H}]$. In this sense, our results can basically fit the observations. More explorations of metal-poor Nrich objects are needed.

We now follow Sect. 5.11 and calculate the contribution by massive stars to nitrogen using the model VG+WW. Figure 10d shows the fractional contribution as a function of the Galactic age. We note that, except at the extreme early stage before the intermediate mass stars began to evolve, the relative contribution from massive stars is very low in the early stage, and that it gradually increases with the evolution of the Galaxy, then slightly decreases in the late stage. This trend means that the yield of nitrogen varies strongly with the metallicity. However, the proportion of nitrogen contributed by massive stars is generally very low, even the peak is less than $20 \%$. WW calculated a very low $\mathrm{N}$ yield for stars with zero metallicity, on the order of $10^{-6} M_{\odot}$. The $\mathrm{N}$ yields of ILMS with low metallicities are higher than WW, for example, the newly formed and ejected nitrogen from $8 M_{\odot}$ stars with $Z=0.001$ is about $10^{-2} M_{\odot}$, thus, the intermediate mass stars have been making important contributions since they began to evolve and eject materials into the ISM. With increase of metallicity, the $\mathrm{N}$ yield of massive stars also increases, as is shown by rising part of the curve of Fig. 10d. The eventual decrease in the curve at the late stage is probably caused by the higher $\mathrm{N}$ yield from the ILMS with higher metallicities.

\section{Oxygen abundance}

The source of oxygen is generally clear, namely, it is produced mainly in short-lived, massive stars and ejected through SNII explosions. As our Galaxy evolves, more and more Fe is produced through long lifetime SN Ia explosions, and the ratio $[\mathrm{O} / \mathrm{Fe}]$ decreases. This trend is shown in all of our calculated results given in Sect. 5 .

Conti et al. (1967) presented the first data indicating the existence of an oxygen overabundance in metalpoor stars. Many authors continued to explore the evolution of $[\mathrm{O} / \mathrm{Fe}]$, and confirmed the overabundance of oxygen in metal-poor stars (see Sect. 2). Edvardsson et al. (1993) and Chen et al. (2000) separately obtained the 

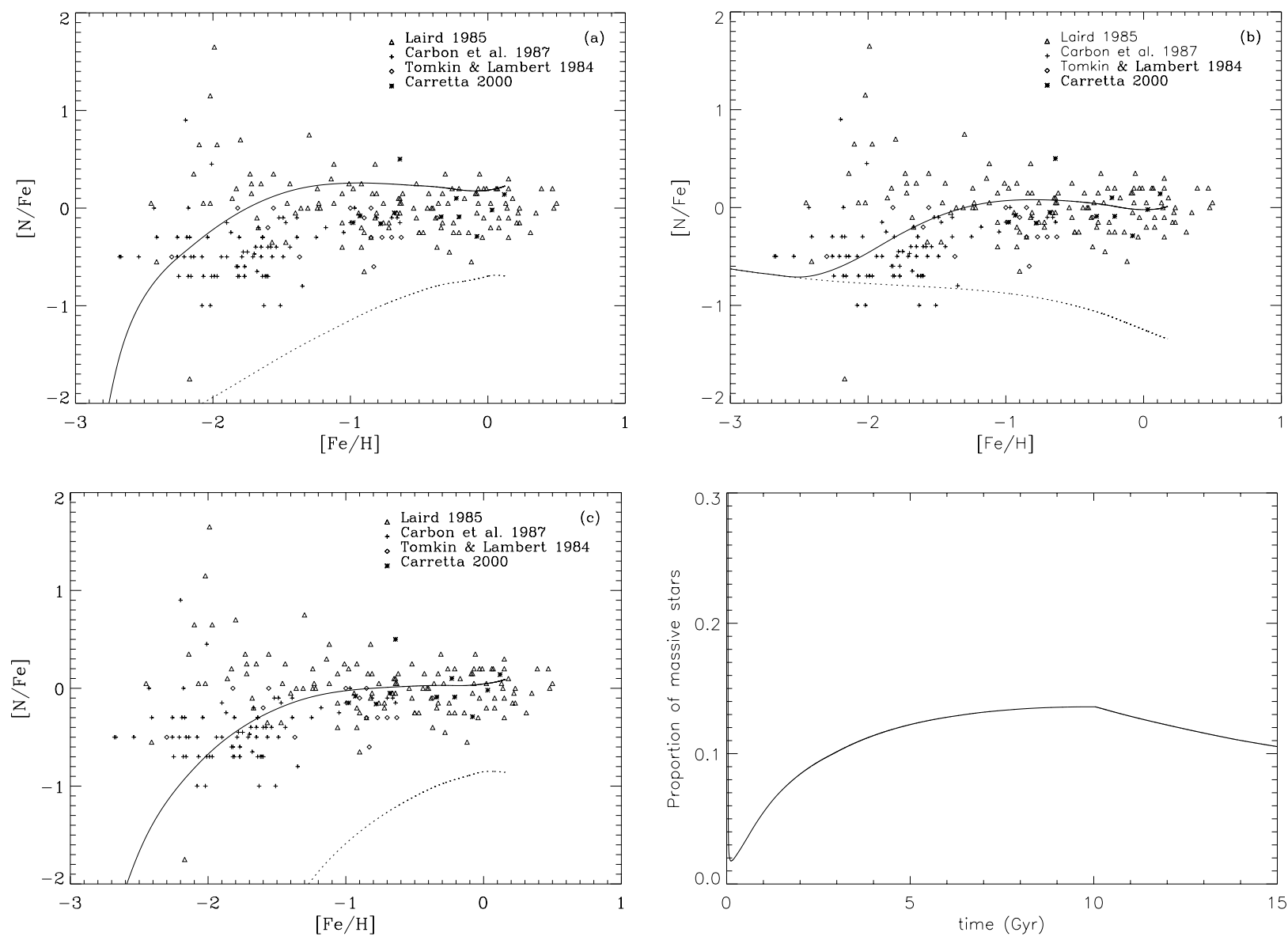

Fig. 10. a) The calculated $[\mathrm{N} / \mathrm{Fe}]$ vs. $[\mathrm{Fe} / \mathrm{H}]$ relation using nucleosynthesis yields of VG for ILMS and WW for massive stars. The solid line represents the result with both the contributions of ILMS and massive stars, and the dashed line represents the result with only massive stars. b) Same as a) but with the yields of N97 for massive stars. c) Same as a) but with the yields of M92 for massive stars. d) The relative contribution of massive stars to nitrogen production with the Galactic ages predicted by using the yields of VG for ILMS and WW for massive stars.

oxygen abundances of a large sample of disk stars, both showed that $[\mathrm{O} / \mathrm{Fe}]$ decreases with increasing metallicity in Galactic disk stars. For halo stars, however, available observations exhibit an apparent contradiction, namely, the $[\mathrm{O} / \mathrm{Fe}]$ values of metal-poor giants derived from the [O I] forbidden line $(+0.4)$ are lower than those of dwarfs from the O I triplet at $\lambda 7770 \AA$ and $\mathrm{OH}$ lines $(+1.0)$ (see Fig. 11). And using the $\lambda 6300 \AA$ forbidden line, similar abundance values to the giants have been obtained for some metal-poor dwarfs, though the line is very weak in these stars (Nissen et al. 2000).

The difference can be understood from two aspects. In the first aspect, we believe that oxygen has a similar behavior to other $\alpha$ elements $(\mathrm{Mg}, \mathrm{Si}, \mathrm{S}, \mathrm{Ca})$, that is, we believe that the lower $[\mathrm{O} / \mathrm{Fe}]$ in metal-poor region is true, and the relatively higher ratio derived from the $\mathrm{O}$ I triplet or $\mathrm{OH}$ line is wrong. The reasons are as follows. The $\mathrm{O}$ I triplet has a very high excitation feature $(9.15 \mathrm{eV})$ and is quite sensitive to the temperature structure of the adopted model atmosphere, and NLTE effects cause the higher $\mathrm{O}$ abundance derived from it. And the $\mathrm{OH}$ lines in the UV are very sensitive to the temperature structure of the stellar atmosphere and/or to NLTE ef- fects too. According to this argument, there should be no obvious change in the $\mathrm{O}$ abundance as the dwarfs evolve to the giants.

On the other hand, if we believe that the higher $[\mathrm{O} / \mathrm{Fe}]$ derived from the $\mathrm{O}$ I triplet and $\mathrm{OH}$ line reflects the true O abundance, then we have to reconsider the abundance evolution of oxygen, hence the GCE result. Maybe the top-heavy IMF is true, which favours the formation of massive stars in the early Galactic stage, hence a high O abundance. This assumption would imply a corresponding high $[\alpha / \mathrm{Fe}]$ in metal-poor dwarfs, which is not found in present available data. Could the difference between $[\mathrm{O} / \mathrm{Fe}]$ and $[\alpha / \mathrm{Fe}]$ mean that their behaviors are not similar? Perhaps NLTE effects can revise the present $[\alpha / \mathrm{Fe}]$ value. Recently, Zhao \& Gehren (2000) reported that NLTE effects can increase the $[\mathrm{Mg} / \mathrm{Fe}]$ abundance, and the corrections are larger for the lower metallicities of the metal-poor stars. Thus, maybe NLTE corrections can also increase the abundances of other $\alpha$ elements at the early Galactic stage.

Goswami \& Prantzos (2000) pointed out three reasons for the higher $[\mathrm{O} / \mathrm{Fe}]$ value in low metallicity region: (1) The Fe produced by SN Ia explosions entered the 


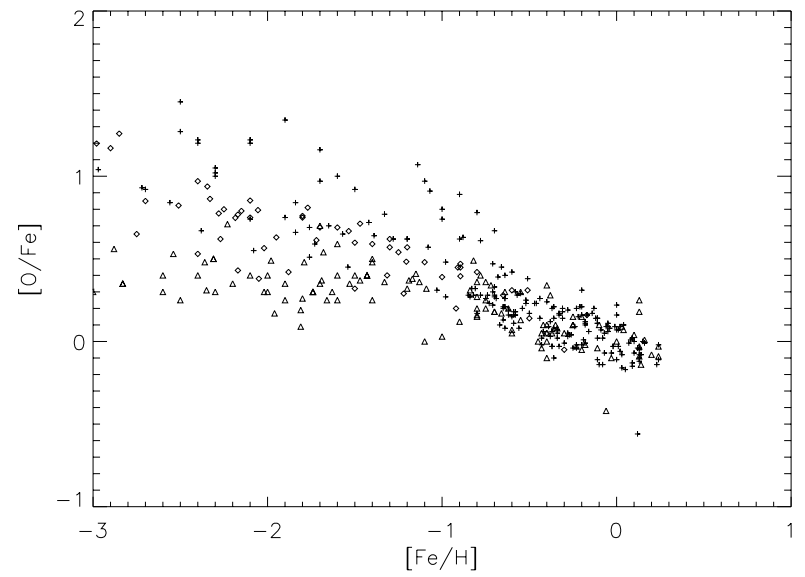

Fig. 11. The observed $[\mathrm{O} / \mathrm{Fe}]$ vs. $[\mathrm{Fe} / \mathrm{H}]$ data. Oxygen abundances derived from $[\mathrm{O}$ I] $6300 \AA$ forbidden line are indicated by triangles, from $\mathrm{O}$ I triplet by pluses, and from $\mathrm{OH}$ lines in UV region by diamonds.

galactic scene as early as $[\mathrm{Fe} / \mathrm{H}] \sim-3$, instead of $[\mathrm{Fe} / \mathrm{H}] \sim$ -1 in the "standard" scenario; $(2)$ the $\mathrm{O}$ yields from massive stars are, for some reasons, metallicity dependent; (3) there is the possibility of the yields of Fe and all elements heavier than oxygen being metallicity-dependent.

\section{Conclusions}

Using the standard GCE model, we have attempted an exploration of the questions of the sources of carbon and the abundance evolution of CNO elements. Because the yield of stellar nucleosynthesis is a very important parameter of the GCE model, and different yields give different evolutionary behaviors of the elements, we chose the recently published different nucleosynthesis yields: the yields given by RV, VG, MBC and M2K for intermediate-, low-mass single stars; the results of the classical W7 model of Nomoto et al. (1997b) for binary stars through SN Ia explosions; the yields given by WW, N97, M92 and PCB for massive single stars. Then we set up 8 specific models for the combinations, $\mathrm{VG}+\mathrm{WW}, \mathrm{VG}+\mathrm{N} 97$, $\mathrm{VG}+\mathrm{M} 92, \mathrm{RV}+\mathrm{WW}, \mathrm{RV}+\mathrm{N} 97, \mathrm{RV}+\mathrm{M} 92, \mathrm{MBC}+\mathrm{PCB}$ and $\mathrm{M} 2 \mathrm{~K}+\mathrm{PCB}$.

Our results show that, after fitting the predicted agemetallicity relation, metallicity distribution of $\mathrm{G}$ dwarfs and $[\mathrm{O} / \mathrm{Fe}]$ vs. $[\mathrm{Fe} / \mathrm{H}]$ relation to the observations, very different $[\mathrm{C} / \mathrm{Fe}]$ vs. $[\mathrm{Fe} / \mathrm{H}]$ relations are given by the different models with different yield combinations.

The ${ }^{12} \mathrm{C}(\alpha, \gamma){ }^{16} \mathrm{O}$ reaction rate is very important to stellar nucleosynthesis calculations. Hence, it affects the results of the GCE calculations. A higher rate of this reaction will produce less carbon, and a lower rate, more. A suitable choice is between the values given by $\mathrm{CFHZ}$ and $\mathrm{CF}$.

Generally speaking, in the early stage of our Galaxy, massive stars are the main contributor of carbon. As our Galaxy evolves to the late stage, the long lifetime ILMS begins to play an important role in the enrichment of the ISM. At the same time, the high metallicity W$\mathrm{R}$ stars eject significant amounts of carbon into the ISM by radiative-driven stellar wind; the metallicity influences the outer opacities and therefore the mass loss rate by stellar winds in massive stars. However, on the present published nucleosynthesis yields, our results still cannot distinguish the source of carbon in metal-rich Galactic stage. It may be that just the massive stars $\left(M>8 M_{\odot}\right)$ are sufficient to account for the observed $[\mathrm{C} / \mathrm{Fe}]$ values (see Figs. 3e, 6e); or it may be just the ILMS and $M \leq 40 M_{\odot}$ massive stars without the $\mathrm{W}-\mathrm{R}$ stars are enough to explain the observed carbon in metal-rich stars (see Figs. 1b, 6f, 7f).

However, we can at least distinguish the different contributions of ILMS and massive stars to carbon from our calculations. Having comparing the GCE results shown in Sects. 5.1-5.8, and considered the undetermined characters of stellar wind mass loss of massive stars, and assuming the actual ${ }^{12} \mathrm{C}(\alpha, \gamma){ }^{16} \mathrm{O}$ reaction rate to be between $\mathrm{CFHZ}$ and $\mathrm{CF}$, we calculated the respective contributions to carbon from massive stars and ILMS in the model WW+VG. Figure 9 shows that, in the early stage, almost all carbon is produced by massive stars; with increase of the Galactic age, the contribution from ILMS becomes more and more and eventually exceeds that of the massive stars. The latter contribution is increased when the contribution from W-R stars is included.

Henry et al. (2000) gave an excellent analysis on the source of $\mathrm{C}$ and N. But they only gave detailed results for the yields of VG for ILMS and M92 for massive stars. And they increased the yields of M92 in their calculations (see their Table 3), which increased the contribution of carbon from massive stars.

Carigi (2000) compared different set of yield, and suggested that massive stars with stellar wind are the main source of carbon. Compared to their work, we use the abundances of a large sample of dwarf stars as one of the most important observational constraints rather than just the stellar age. The reason is that few halo stars have determined ages, while the results reported by Edvardsson et al. (1993), Gustafsson et al. (1999) and Chen et al. (2000) are only for disk stars, so the element abundance in the early stage of the Galaxy cannot be tested by observations. In addition, we divided the massive stars into two ranges, $M \leq 40 M_{\odot}$ and $M>40 M_{\odot}$, so that we can compare the carbon contributions given by W-R stars and ILMS more clearly. And we use the new set of nucleosynthesis yields of M2K, which contain important information on low metallicity ILMS.

Our results confirm that most of nitrogen is produced by ILMS, especially by intermediate mass stars. And the present yields given by massive stars cannot supply the needed primary $\mathrm{N}$ source. Massive stars produce the main part of oxygen in the universe. But the observed $\mathrm{O}$ abundances derived from different atomic lines lead to obvious differences in the $[\mathrm{O} / \mathrm{Fe}]$ determination in metal-poor stars. Maybe we should check the procedure and many corresponding parameters to obtain abundance of oxygen. Also, 
perhaps this difference hints at some new information on Galactic chemical evolution, especially, it is necessary to understand the evolution of our Galaxy in the early stage carefully.

Acknowledgements. We thank Dr. B. Edvardsson for very valuable suggestions on the original manuscript. We thank Dr. T. Kiang and Dr. P. Podsiadlowski for spending much time and energy to help us to improve the English expression. Thank Dr. L. C. Deng for the interesting discussions about massive stars, and thank Zhang Yanxia and Fan Yingjie for their friendly help. This research work is supported by the National Natural Science Foundation of China under grant No. 19725312 and NKBRSF G1999075406.

\section{References}

Abia, C., \& Rebolo, R. 1989, ApJ, 347, 186

Andersson, H., \& Edvardsson, B. 1994, A\&A, 290, 590

Balachandran, S. C., \& Carney, B. W. 1996, AJ, 111, 946

Barbuy, B. 1988, A\&A, 191, 121

Barbuy, B., \& Erdelyi-Mendes, M. 1989, A\&A, 214, 239

Beveridge, C. R., \& Sneden, C. 1994, AJ, 108, 285

Boesgaard, A. M., \& King, J. R. 1993, AJ, 106, 2309

Boesgaard, A. M., King, J. R., Deliyannis, C. P., \& Vogt, S. S. 1999, AJ, 117, 492

Burbidge, E. M., Burbidge, G. R., Fowler, W. A., \& Hoyle, F. 1957, Rev. Mod. Phys., 29, 547

Carbon, D. F., Barbuy, B., Kraft, R. P., Friel, E. D., \& Suntzeff, N. B. 1987, PASP, 99, 335

Carigi, L. 1994, ApJ, 424, 181

Carigi, L. 2000, Rev. Mex. Astron. Astrofis. 36 [astro-ph/0005042]

Carlberg, R. G., Dawson, P. C., Hsu, T., \& VandenBerg, D. A. 1985, ApJ, 294, 674

Carretta, E., Gratton, R. G., \& Sneden, C. 2000, A\&A, 356, 238

Caughlan, G. R., Fowler, W. A., Harris, M. J., \& Zimmerman, B. A. 1985, At. Data Nucl. Data Tables, 32, 197 (CFHZ)

Caughlan, G. R., \& Fowler, W. A. 1988, At. Data Nucl. Data Tables, 40, $283(\mathrm{CF})$

Chang, R. X., Hou, J. L., Shu, C. G., \& Fu, C. Q. 1999, A\&A, 350,38

Chen, Y. Q., Nissen, P. E., Zhao, G., Zhang, H. W., \& Benoni, T. 2000, A\&AS, 141, 491

Chiappini, C., Matteucci, F., \& Gratton, R. 1997, ApJ, 477, 765

Chiappini, C., Matteucci, F., \& Padoan, P. 2000, ApJ, 528, 711

Chieffi, A., Limongi, M., \& Straniero, O. 1998, ApJ, 502, 737

Clegg, R. E. S., Lambert, D. L., \& Tomkin, J. 1981, ApJ, 250, 262

Conti, P. E. 1995, in The 11th IAP Astrophysics Meeting, Proc. The Interplay Between Massive Star Formation, the ISM and Galaxy Evolution, ed. D. Kunth, B. Guiderdoni, M. Heydari-Malayeri, T. X. Thuan (Paris: Éditions Frontières), 37

Conti, P. S., Greenstein, J. L., Spinard, H., Wallerstein, G., \& Vardya, M. S. 1967, ApJ, 148, 105

Crowther, P. A., 2000a, in Proc. The Influence of Binaries on Stellar Population Studies, ed. D. Vanbeveren (Kluwer) [astro-ph/0010581]
Crowther, P. A. 2000b, A\&A, 356, 191

Dopita, M. A., \& Ryder, S. D. 1994, ApJ, 430, 163

Dwek, E. 1998, ApJ, 501, 643

Edvardsson, B., Andersen, J., Gustafsson, B., et al. 1993, A\&A, 275,101

Forestini, M., \& Charbonnel, C. 1997, A\&AS, 123, 241

Fuhrmann, K. 1998, A\&A, 338, 161

Fulbright, J. P., \& Kraft, R. P. 1999, ApJ, 118, 527

Garnett, D. R., Shields, G. A., Peimbert, M., et al. 1999, ApJ, 513,168

Grevesse, N., \& Sauval, A. J. 1998, Space Sci. Rev., 85, 161

Goswami, A., \& Prantzos, N. 2000, A\&A, 359, 151

Gratton, R. G., \& Ortolani, S. 1986, A\&A, 169, 201

Gustafsson, B., Karlsson, T., Olsson, E., Edvardsson, B., \& Ryde, N. 1999, A\&A, 342, 426

Gustafsson, B., \& Ryde, N. 2000, in IAU Symp. 177, The carbon Star Phenomenon, ed. R. F. Wing (Kluwer, Dordrecht), 481

Henry, R. B. C., Edmunds, M. G., \& Köppen, J. 2000, ApJ, 541,660

Hou, J. L., Prantzos, N., \& Boissier, S. 2000, A\&A, 362, 921

Iben, I. Jr., \& Truran, J. W. 1978, ApJ, 220, 980

Israelian, G., García López, R. J., \& Rebolo, R. 1998, ApJ, 507,805

Karlsson, T., Edvardsson, B., Gustafsson, B., Olsson, E., \& Ryde, N. 1999, Ap\&SS, 265, 261

King, J. R. 1993, AJ, 106, 1206

King, J. R. 1994, ApJ, 436, 331

King, J. R., \& Boesgaard, A. M. 1995, AJ, 109, 383

Kiselman, D. 1991, A\&A, 245, L9

Kobulnicky, H. A., \& Skillman, E. D. 1998, ApJ, 497, 601

Kroupa, P., Tout, C. A., \& Gilmore, G. 1993, MNRAS, 262, 545

Kudritzki, R-.P., \& Puls, J. 2000, ARA\&A, 38, 613

Laird, J. B. 1985, ApJ, 289, 556

Leitherer, C. 1995, in Proc. The 11th IAP Astrophysics Meeting, The Interplay Between Massive Star Formation, the ISM and Galaxy Evolution, ed. D. Kunth, B. Guiderdoni, M. Heydari-Malayeri, T. X. Thuan (Paris: Éditions Frontières), 27

Langer, N. 1989, A\&A ,220, 135

Liang, Y. C., \& Zhao, G. 2001, Progress in Natural Science, in press

Limongi, M., Straniero, O., \& Chieffi, A. 2000, ApJS, 129, 625

Maciel, W. J. 2000, Rev. Mex. Astron. Astrofis. SC [astro/ph-0012179]

Maeder, A. 1992, A\&A, 264, 105 (M92)

Maeder, A., \& Conti, P. E. 1994, ARA\&A, 32, 227

Marigo, P. 2001, A\&A, 370, 194 (M2K)

Marigo, P., Bressan, A., \& Chiosi, C. 1996, A\&A, 313, 545 (MBC1)

Marigo, P., Bressan, A., \& Chiosi, C. 1998, A\&A, 331, 564 (MBC2)

Martinelli, A., \& Matteucci, F. 2000, A\&A, 353, 269

Massey, P., Lang, C. C., DeGioia-Eastwood, K., \& Garmany, C. D. 1995, ApJ, 438, 188

Matteucci, F., \& François, P. 1989, MNRAS, 239, 885

Meusinger, H., Reimann, H.-G., \& Stecklum, B. 1991, A\&A, 245,57

Meynet, G., \& Maeder, A. 2000, A\&A, 361, 101

Mishenina, T. V., Korotin, S. A., Klochkova, V. G., \& Panchuk, V. E. 2000, A\&A, 353, 978 
Nissen, P. E., \& Edvardsson, B. 1992, A\&A, 261, 255

Nissen, P. E., Gustafsson, B., Edvardsson, B., \& Gilmore, G. 1994, A\&A, 285, 440

Nissen, P. E., Primas, F., \& Asplund, M. 2000, 24th meeting of the IAU, Joint Discussion 8, Oxygen Abundances in Old Stars and Implications to Nucleosynthesis and Cosmology ( Manchester, UK)

Nomoto, K., Hashimoto, M., Tsujimoto, T., et al. 1997a, Nucl. Phys., A616, 79c (N97)

Nomoto, K., Iwamoto, K., Nakasato, N., et al. 1997b, Nucl. Phys., A621, 467c

Oberhummer, H., Csótó, A., \& Schlattl, H. 2000, Science, 289, 88

Pagel, B. E. J. 1997, Nucleosynthesis and Chemical Evolution of Galaxies (Cambridge University Press), 206

Portinari, L., Chiosi, C., \& Bressan, A. 1998, A\&A, 334, 505 (PCB)

Prantzos, N., \& Silk, J. 1998, ApJ, 507, 229

Prantzos, N., Vangioni-Flam, E., \& Chauveau, S. 1994, A\&A, 285,132

Renzini, A., \& Voli, M. 1981, A\&A, 94, 175 (RV)

Sackett, P. D. 1997, ApJ, 483, 103

Salpeter, E. E. 1955, ApJ, 121, 161

Samland, M. 1998, ApJ, 496, 155

Scalo, J. M. 1986, Fund. Cosmic Phys., 11, 1

Schaller, G., Schaerer, D., Meynet, G., \& Maeder, A. 1992, A\&AS, 96, 269

Schmidt, M. 1959, ApJ, 129, 243
Schmutz, W. 1997, A\&A, 321, 268

Spiesman, W. J., \& Wallerstein, G. 1991, AJ, 102, 1790

Spite, M., \& Spite, F. 1991, A\&A, 252, 689

Sneden, C., Kraft, R. P., Prosser, C. F., \& Langer, G. E. 1991, AJ, 102, 2001

Sugimoto, D., \& Nomoto, K. 1980, Space Sci. Rev., 25, 155

Takeda, Y. 1994, PASJ, 46, 53

Talbot, Jr. R. J., \& Arnett, W. D. 1975, A\&A, 197, 551

Thielemann, F.-K., Nomoto, K., \& Hashimoto, M. 1996, ApJ, 460, 408

Thomas, D., Greggio, L., \& Bender, R. 1998, MNRAS, 296, 119

Timmes, F. X., Woosley, S. E., \& Weaver, T. A. 1995, ApJS, 98,617

Tomkin, J., Lemke, M., Lambert, D. L., \& Sneden, C. 1992, AJ, 104, 1568

Tomkin, J., \& Lambert, D. L. 1984, ApJ, 279, 220

Tomkin, J., Sneden, C., \& Lambert, D. L. 1986, ApJ, 302, 415

Tomkin, J., Woolf, V. M., Lambert, D. L., \& Lemke, M. 1995, AJ, 109, 2204

Van den Hoek, L. B., \& Groenewegen, M. A. T. 1997, A\&AS, $123,305(\mathrm{VG})$

Wheeler, J. C., Sneden, C., \& Truran, Jr. J. W. 1989, ARA\&A, 27, 279

Whelan, J., \& Iben, I. Jr. 1973, ApJ, 186, 1007

Woosley, S. E., \& Weaver, T. A. 1995, ApJS, 101, 181 (WW)

Zhao, G., \& Gehren, T. 2000, A\&A, 362, 1077

Zhao, G., \& Magain, P. 1990, A\&A, 238, 242 